\title{
COMPOSITION ALGEBRAS, EXCEPTIONAL JORDAN ALGEBRA AND RELATED GROUPS*
}

\section{IVAN TODOROV AND SVETLA DRENSKA}

Communicated by Ivaïlo M. Mladenov

\begin{abstract}
Normed division rings are reviewed in the more general framework of composition algebras that include the split (indefinite metric) case. The Jordan von Neumann - Wigner classification of finite dimensional Jordan algebras is outlined with special attention to the 27 dimensional exceptional Jordan algebra $\mathfrak{J}$. The automorphism group $\mathrm{F}_{4}$ of $\mathfrak{J}$ and its maximal Borel-de Siebenthal subgroups $\frac{\mathrm{SU}(3) \times \mathrm{SU}(3)}{\mathbb{Z}_{3}}$ and $\operatorname{Spin}(9)$ are studied in some detail with an eye to possible applications to the fundamental fermions in the Standard Model of particle physics.
\end{abstract}

MSC: 20G41, 17C40, 117A75, 7C60, 17A35, 22D20, 22E15, 22E20, 22E46

Keywords: Exceptional Lie groups, Jordan algebra, octonions, standard model

\section{Contents}

$\begin{array}{llr}1 & \text { Introduction } & \mathbf{6 0}\end{array}$

2 Composition and Clifford Algebras $\quad 60$

2.1 Normed Alternative Algebras . . . . . . . . . . . . . . . . . . . . 60

2.2 Relation to Clifford Algebras and Classification . . . . . . . . . . . . . . 63

2.3 Historical Note . . . . . . . . . . . . . . . . . . 66

3 Octonions. Isometries and Automorphisms $\quad 67$

3.1 Eight Dimensional Alternative Algebras . . . . . . . . . . . . . . . . 67

3.2 Isometry Group of the (Split) Octonions. Triality . . . . . . . . . . . . 68

3.3 Automorphism Group and Derivations of Octonions . . . . . . . . . . . 69

3.4 Roots and Weights of $\mathfrak{g}_{2(2)} \subset \mathfrak{s o}(4,4) \ldots \ldots \ldots 71$

4 Jordan Algebras and Related Groups $\quad \mathbf{7 3}$

4.1 Classification of Finite Dimensional Jordan Algebras . . . . . . . . . . 73

4.2 Automorphism Groups of the Exceptional Jordan Algebras $\mathcal{H}_{3}\left(\mathbb{O}_{(s)}\right)$ and their Maximal Subgroups . . . . . . . . . . . . . . 76

4.3 The Jordan Subalgebra $\operatorname{JSpin}_{9}$ of $\mathcal{H}_{3}(\mathbb{O})$ and its Automorphism Group $\operatorname{Spin}(9) \subset \mathrm{F}_{4} \ldots \ldots \ldots \ldots \ldots \ldots \ldots$

${ }^{*}$ Dedicated to the memory of Professor Vasil V. Tsanov 1948-2017. 
4.4 The Structure Group $\operatorname{Spin}(9,1)$ of $\mathrm{JSpin}_{9}$ and its 32-Dimensional Dirac Spinor Representation . . . . . . . . . . . . . . . . . . . . 83

5 The Symmetry Algebra of the Standard Model 85

$\begin{array}{llr}6 & \text { Outlook } & 87\end{array}$

$\begin{array}{ll}\text { References } & 91\end{array}$

\section{Introduction}

Division and Clifford algebras were introduced in 19th century with an eye for applications in geometry and physics (for a historical survey see the last chapter of [24]). Pascual Jordan introduced and studied his algebras in the 1930's in order to describe observables in quantum mechanics (for a taste of Jordan algebras see [25] along with the original paper [23]). Yet, the first serious applications of these somewhat exotic structures appeared (in mid-twentieth century) in pure mathematics: in the theory of exceptional Lie groups and symmetric spaces (cf. $[17,19]$ ) as well as the later surveys $[1,5,26]$ in topology [2]. Possible applications to particle physics were first advocated by Feza Gürsey and his students in the 1970's - see his lecture and his posthumous book (with C.-H.Tze) [21] and references therein). They continue in various guises to attract attention until these days, never becoming a mainstream activity. The present lectures are meant as a background for the ongoing work $[14,30]$. Although this proposal of an "exceptional quantum geometry" is still tentative, we feel that it is worth pursuing. ${ }^{1}$ In any case, the mathematical background which is the main subject of these notes is sound and beautiful - and deserves to be known by particle theorists.

\section{Composition and Clifford Algebras}

\subsection{Normed Alternative Algebras}

A composition (or Hurwitz) algebra $\mathcal{A}$ is a vector space over a field $\mathbb{K}=(\mathbb{R}, \mathbb{C}, \ldots)$ equipped with a bilinear (not necessarily associative) product $x y$ with a unit 1 $(1 x=x 1=x)$ and a nondegenerate quadratic form $N(x)$, the norm satisfying

$$
N(x y)=N(x) N(y), \quad N(\lambda x)=\lambda^{2} N(x) \quad \text { for } \quad x \in \mathcal{A}, \quad \lambda \in \mathbb{K} .
$$

\footnotetext{
${ }^{1}$ For related attempts to provide an algebraic counterpart of the Standard Model of particle physics see $[8,11-13,18,28]$ and references to earlier work cited therein.
} 
The norm allows to define by polarization a symmetric bilinear form $\langle x, y\rangle$ setting

$$
2\langle x, y\rangle=N(x+y)-N(x)-N(y)=2\langle y, x\rangle .
$$

Nondegeneracy of $N$ means that if $\langle x, y\rangle=0$ for all $y \in \mathcal{A}$ then $x=0$. By repeated polarization of the identity $\langle x y, x y\rangle=\langle x, x\rangle\langle y, y\rangle$ one obtains

$$
\begin{gathered}
\langle a b, a c\rangle=N(a)\langle b, c\rangle=\langle b a, c a\rangle \\
\langle a c, b d\rangle+\langle a d, b c\rangle=2\langle a, b\rangle\langle c, d\rangle .
\end{gathered}
$$

Setting in (4) $a=c=x, b=1, d=y$ and using (3) we find

$$
\left\langle x^{2}+N(x) 1-t(x) x, y\right\rangle=0
$$

where $t(x):=2\langle x, 1\rangle$ is by definition the trace, or, using the non-degeneracy of the form $\langle\cdot, \cdot\rangle$

$$
x^{2}-t(x) x+N(x) 1=0, \quad t(x)=2\langle x, 1\rangle .
$$

Thus every $x \in \mathcal{A}$ satisfies a quadratic relation with coefficients the trace $t(x)$ and the norm $N(x)$ (a linear and a quadratic scalar functions) taking values in $\mathbb{K}$.

The trace functional (5) allows to introduce Cayley conjugation

$$
x \rightarrow x^{*}=t(x)-x, \quad t(x)=t(x) 1 \in \mathcal{A}
$$

an important tool in the study of composition algebras. It is an (orthogonal) reflection $\left(\left\langle x^{*}, y^{*}\right\rangle=\langle x, y\rangle\right)$ that leaves the scalars $\mathbb{K} 1$ invariant (in fact, $t(\lambda 1)=2 \lambda$ implying $(\lambda 1)^{*}=\lambda 1$ for $\left.\lambda \in \mathbb{K}\right)$. It is also an involution and an antihomomorphysm

$$
\left(x^{*}\right)^{*}=x, \quad(x y)^{*}=y^{*} x^{*} .
$$

Furthermore equations (5) and (6) allow to express the trace and the norm as a sum and a product of $x$ and $x^{*}$

$$
t(x)=x+x^{*}, \quad N(x)=x x^{*}=x^{*} x=N\left(x^{*}\right) .
$$

The relation (4) allows to deduce

$$
\langle a x, y\rangle=\left\langle x, a^{*} y\right\rangle, \quad\langle x a, y\rangle=\left\langle x, y a^{*}\right\rangle .
$$

From these identities it follows $\langle a b, 1\rangle=\left\langle a, b^{*}\right\rangle=\langle b a, 1\rangle$, hence, the trace is commutative

$$
t(a b)=\left\langle b, a^{*}\right\rangle=\left\langle a, b^{*}\right\rangle=t(b a) .
$$


Similarly, one proves that $t$ is associative and symmetric under cyclic permutations

$$
t((a b) c)=t(a(b c))=: t(a b c)=t(c a b)=t(b c a) .
$$

Moreover, using the quadratic relation (5) and the above properties of the trace one proves the identities that define an alternative algebra

$$
x^{2} y=x(x y), \quad y x^{2}=(y x) x
$$

(see [26, Section 1] for details). The conditions (11) guarantee that the associator

$$
[x, y, z]=(x y) z-x(y z)
$$

changes sign under odd permutations (and is hence preserved by even, cyclic, permutations). This implies, in particular, the flexibility conditions

$$
(x y) x=x(y x) .
$$

An unitar alternative algebra with an involution $x \rightarrow x^{*}$ satisfying (7) is a composition algebra if the norm $N$ and the trace $t$ defined by (9) are scalars (i.e., belong to $\mathbb{K}(=\mathbb{K} 1)$ ) and the norm is non-degenerate.

Given a finite dimensional composition algebra $\mathcal{A}$ Cayley and Dickson have proposed a procedure to construct another composition algebra $\mathcal{A}^{\prime}$ with twice the dimension of $\mathcal{A}$. Each element $x$ of $\mathcal{A}^{\prime}$ is written in the form

$$
x=a+e b, \quad a, b \in \mathcal{A}
$$

where $e$ is a new "imaginary unit" such that

$$
e^{2}=-\mu, \quad \mu \in\{1,-1\} .
$$

Thus $\mathcal{A}$ appears as a subalgebra of $\mathcal{A}^{\prime}$. The product of two elements $x=a+e b$, $y=c+e d$ of $\mathcal{A}^{\prime}$ is defined as

$$
x y=a c-\mu d \bar{b}+e(\bar{a} d+c b)
$$

where $a \rightarrow \bar{a}$ is the Cayley conjugation in $\mathcal{A}$. (The order of the factors becomes important, when the product in $\mathcal{A}$ is noncommutative.) The Cayley conjugation $x \rightarrow x^{*}$ and the norm $N(x)$ in $\mathcal{A}^{\prime}$ are defined by

$$
\begin{aligned}
x^{*} & =(a+e b)^{*}=\bar{a}+\bar{b} e^{*}=\bar{a}-\bar{b} e=\bar{a}-e b \\
N(x) & =x x^{*}=a \bar{a}+\mu b \bar{b}=x^{*} x .
\end{aligned}
$$

Let us illustrate the meaning of (16) and (17) in the first simplest cases. 
For $\mathcal{A}=\mathbb{R}, \bar{a}=a,(16)$ coincides with the definition of complex numbers for $\mu=1(e=\mathrm{i})$ and defines the split complex numbers for $\mu=-1$. Taking next $\mathcal{A}=\mathbb{C}$ and $\mu=1$ we can identify $\mathcal{A}^{\prime}$ with a $2 \times 2$ matrix representations setting

$$
\begin{aligned}
& \mathbf{a}=a_{0}+e_{1} a_{1}=a_{0}+\mathrm{i} \sigma_{3} a_{1}=\left(\begin{array}{ll}
a & 0 \\
0 & \bar{a}
\end{array}\right), \quad a=a_{0}+\mathrm{i} a_{1} \\
& x=\mathbf{a}+e \mathbf{b}, \quad e=\left(\begin{array}{rr}
0 & -1 \\
1 & 0
\end{array}\right) \Rightarrow x=\left(\begin{array}{rr}
a & -\bar{b} \\
b & \bar{a}
\end{array}\right), \quad \mathbf{b}=b_{0}+e_{1} b_{1} .
\end{aligned}
$$

Anticipating Baez Fano plane [3] notation for the octonion imaginary units (see Application A) we shall set $e=e_{4}, e_{4} e_{1}=e_{2}\left(=\mathrm{i} \sigma_{1}\right)$.

It is then easily checked that the multiplication law (16) reproduces the standard matrix multiplication, the Cayley conjugation $x \rightarrow x^{*}$ coincides with the hermitian conjugation of matrices, while the norm $N(x)$ in $\mathcal{A}^{\prime}$ is given by the determinant

$$
\mathbb{H}=\left\{x \in \mathbb{C}[2] ; x x^{*}=\operatorname{det} x(\geq 0)\right\} .
$$

Similarly, starting with the split complex numbers, we can write

$\mathbf{a}_{\mathbf{s}}=\mathbf{a}_{\mathbf{0}}+\tilde{\mathbf{e}}_{1} \mathbf{a}_{1}, \quad \tilde{\mathbf{e}}_{1}=\sigma_{3} \leftrightarrows \mathbf{a}_{\mathbf{s}}=\left(\begin{array}{cc}a_{s} & 0 \\ 0 & \bar{a}_{s}\end{array}\right), \quad \mathbf{a}_{\mathbf{s}}=\mathbf{a}_{\mathbf{0}}+\mathbf{a}_{1}, \quad \overline{\mathbf{a}}_{\mathbf{s}}=\mathbf{a}_{\mathbf{0}}-\mathbf{a}_{\mathbf{1}}$

and choosing the same $e$ as above we can identify the split quaternions $\mathbb{H}_{s}$ with real $2 \times 2$ matrices

$$
\mathbb{H}_{s}=\left\{x=\left(\begin{array}{cc}
a_{s} & -\bar{b}_{s} \\
b_{s} & \bar{a}_{s}
\end{array}\right) \in \mathbb{R}[2] ; x^{*}=\left(\begin{array}{rr}
\bar{a}_{s} & \bar{b}_{s} \\
-b_{s} & a_{s}
\end{array}\right), x x^{*}=\operatorname{det} x\right\}
$$

its norm having signature $(2,2)$.

The next step in Cayley-Dickson construction gives the octonions, which have a nonassociative (but alternative) multiplication and thus do not have matrix realization.

\subsection{Relation to Clifford Algebras and Classification}

Given a composition algebra $\mathcal{A}$ we define subspace $\mathcal{A}_{0} \subset \mathcal{A}$ of pure imaginary elements with respect to the Cayley conjugation (6)

$$
\mathcal{A}_{0}=\left\{y \in \mathcal{A} ; y^{*}=-y\right\} \text {. }
$$

It is a subspace of co-dimension one, orthogonal to the unit 1 of $\mathcal{A}$. For any $x \in \mathcal{A}$ we define its imaginary part as

$$
x_{0}=\frac{1}{2}\left(x-x^{*}\right)=x-\langle x, 1\rangle \quad \Rightarrow \quad\left\langle x_{0}, 1\right\rangle=0 .
$$


Table 1. The types of algebra $C l(p, q)$ depend on $p-q \bmod 8$.

\begin{tabular}{cccccc}
\hline$n$ & Cliff $_{(1-\mathrm{n})}$ & Irreducible spinor & $n$ & Cliff $_{(1-\mathrm{n})}$ & Irreducible spinor \\
\hline 1 & $\mathbb{R}$ & $S_{1}=\mathbb{R}$ & 5 & $\mathbb{H}[2]$ & $S_{5}=\mathbb{H}^{2}$ \\
2 & $\mathbb{C}$ & $S_{2}=\mathbb{C}$ & 6 & $\mathbb{C}[4]$ & $S_{6}=\mathbb{C}^{4}$ \\
3 & $\mathbb{H}$ & $S_{3}=\mathbb{H}$ & 7 & $\mathbb{R}[8]$ & $S_{7}=\mathbb{R}^{8}$ \\
4 & $\mathbb{H} \oplus \mathbb{H}$ & $S_{4}^{+}=\mathbb{H}, S_{4}^{-}=\mathbb{H}$ & 8 & $\mathbb{R}[8] \oplus \mathbb{R}[8]$ & $S_{8}^{+}=\mathbb{R}^{8}, S_{8}^{-}=\mathbb{R}^{8}$ \\
\hline
\end{tabular}

From the expression $N(x)=x x^{*}$ (8) and from the defining property (21) of imaginary elements it follows that

$$
x_{0} \in \mathcal{A}_{0} \quad \Rightarrow \quad x_{0}^{2}=-N\left(x_{0}\right) .
$$

In other words, if the composition algebra $\mathcal{A}$ is $n$-dimensional then its $(n-1)$ dimensional subalgebra $\mathcal{A}_{0}$ gives rise to a Clifford algebra. If the norm $N$ is positive definite then ${ }^{2} \mathcal{A}_{0}=\operatorname{Cliff}(0, \mathrm{n}-1)=\operatorname{Cliff}_{(1-\mathrm{n})}$. In the case of split complex numbers, quaternions and octonions one encounters instead the algebras Cliff $_{1} \equiv \operatorname{Cliff}(1,0), \operatorname{Cliff}(2,1)$ and $\operatorname{Cliff}(4,3)$, respectively.

It turns out that the classification of the Clifford algebras Cliff $_{(1-n)}$ implies the classification of normed division rings of dimension $n$. So we recall it in the following table: Here we use the notation $\mathbb{A}[n]$ for the algebra $n \times n$ matrices with entries in the (associative) algebra $\mathbb{A}$. As discovered by Elie Cartan in 1908 $\mathrm{Cliff}_{(-\nu-8)}=\mathrm{Cliff}_{-\nu} \otimes \mathbb{R}[16]$ so that the above table suffices to reconstruct all Clifford algebras of type $\mathrm{Cliff}_{-\nu}$. We see that the (real) dimension of the irreducible representation of $\mathrm{Cliff}_{(1-\mathrm{n})}$ coincides with $n$ for $n=1,2,4,8$ only thus implying Hurwitz theorem (see [3, Theorem 1] and the subsequent discussion).

Proceeding to the split alternative composition algebras we note that the type of Cliff $(\mathrm{p}, \mathrm{q})$ only depends on the signature $p-q$ which is 1 (similar to -7 ) for all above cases $\operatorname{Cliff}(1,0)=\mathbb{R} \oplus \mathbb{R}, \operatorname{Cliff}(2,1)=\mathbb{R}[2] \oplus \mathbb{R}[2]$, Cliff $(4,3)=$ $\mathbb{R}[8] \oplus \mathbb{R}[8]$ and

$$
\operatorname{Cliff}(\mathrm{p}, \mathrm{p}-1) \cong \mathbb{R}\left[2^{(\mathrm{p}-1)}\right] \oplus \mathbb{R}\left[2^{(\mathrm{p}-1)}\right] .
$$

All these cases are summarized in Table 1 . We note here the difference in the treatment of the representations of $\operatorname{Cliff}(\mathrm{p}, \mathrm{p}-1)$ in the cases $p=1,2$, in which we are dealing with real associative composition algebras $\mathbb{C}_{s}$ and $\mathbb{H}_{s}$, and $p=4$ of the split octonions. In the associative case we deal with the action of $\operatorname{Cliff}(\mathrm{p}, \mathrm{p}-1)$ on the direct sum $\mathbb{R}^{n} \oplus \mathbb{R}^{n}, n=2^{(p-1)}$ (for $p=1,2$ ) while in the non-associative case it acts on the irreducible subspace $\mathbb{R}^{n}(n=8)$, thus again fitting the dimension of the corresponding alternative algebra.

\footnotetext{
${ }^{2}$ We adopt the sign convention of [24], [21], [29]. The opposite sign convention, Cliff $(n-1)$ for the positive definite $N(x)$, is used e.g. in [3].
} 
Remark 1. The spinors $S_{n}$ are here understood as quantities transforming under the lowest order faithful irreducible representation of the (compact) group $\operatorname{Spin}(n)$ which consists of the norm one even elements of Cliff ${ }_{-n}$. In fact, the even part $\operatorname{Cliff}_{0}(\mathrm{p}, \mathrm{q})$ of $\operatorname{Cliff}(\mathrm{p}, \mathrm{q})$ is isomorphic, for $q>0$ to $\operatorname{Cliff}(\mathrm{p}, \mathrm{q}-1)$. $\operatorname{Spin}(n)$ is the double cover of the rotation group $\mathrm{SO}(\mathrm{n})$. The group of all norm one elements $\mathrm{Cliff}_{(1-\mathrm{n})}$ is the double cover $\mathrm{Pin}(\mathrm{n}-1)$ of the full orthogonal group $\mathrm{O}(\mathrm{n}-1)$ and its irreducible representations are called "pinors" - see [3, Section 2.3].

In summary, the alternative algebras are classified as follows ([26,Proposition 1.6])

Theorem 2. Let $(\mathcal{A}, N)$ be a composition algebra. For $\mu= \pm 1$, denote by $\mathcal{A}(\mu)$ the algebra $\mathcal{A}(\mu)=\mathcal{A} \oplus e \mathcal{A}$ with $e^{2}=-\mu$ and product (16). Then

- $\mathcal{A}(\mu)$ is commutative iff $\mathcal{A}=\mathbb{K}$

- $\mathcal{A}(\mu)$ is associative iff $\mathcal{A}$ is associative and commutative

- $\mathcal{A}(\mu)$ is alternative iff $\mathcal{A}$ is associative.

Theorem 3 ([26], see also the relations (7)-(10)) . A composition algebra is, as a vector space, 1,2, 4 or 8 dimensional. There are four composition algebras $\mathcal{A}_{j}$ over $\mathbb{C}$ of dimension $2^{j}, j=0,1,2,3$. There are seven composition algebras over $\mathbb{R}:$ the division algebras $\mathcal{A}_{j}^{+},(j=0,1,2,3)$ with $N(x) \geq 0$ and $x^{-1}=\frac{x^{*}}{N(x)}$ for $x \neq 0$, and the split algebras $\mathcal{A}_{j}^{s}, j=1,2,3$ of signature $\left(2^{j-1}, 2^{j-1}\right)$.

All above algebras are unique up to isomorphism. The multiplication rule (16) varies in different expositions. Different conventions are related by algebra automorphisms. (Our notation differs from Roos [26] only by the sign of $\mu$, as we set $e^{2}=-\mu$.) The only nontrivial automorphism of the algebra of complex numbers is the complex conjugation. The automorphism group of the (real) quaterions is $\mathrm{SO}(3)$ realized by

$$
x \rightarrow u x u^{*}, \quad u \in \mathrm{SU}(2), \quad \mathrm{u}^{*}=\mathrm{u}^{-1} .
$$

Similarly, the automorphism group of the split quaternions is $\mathrm{SO}(2,1)$

$$
\mathbb{H} \ni x \rightarrow g x g^{-1}, \quad g \in \mathrm{SL}(2, \mathbb{R}) \text {. }
$$

We shall survey the octonions and their automorphisms in the next section. 


\subsection{Historical Note}

The simplest relation of type (1), the one applicable to the absolute value square of a product of complex numbers

$$
(x u-y v)^{2}+(x v+y u)^{2}=\left(x^{2}+y^{2}\right)\left(u^{2}+v^{2}\right)
$$

$(x, y, u, v \in \mathbb{R})$, was found by Diophantus of Alexandria around $250 \mathrm{BC}$. A more general relation of this type

$$
(x u+D y v)^{2}-D(x v+y u)^{2}=\left(x^{2}-D y^{2}\right)\left(u^{2}-D v^{2}\right)
$$

occurs for special values of $D$ in Indian mathematics (cf. Brahmegupta 598 AD) see [6, Section 2]. For $D$ positive it applies to the split complex numbers. The geometric interpretation by Gauss comes much later. (The fact that complex numbers are useful and should be taken seriously is sometimes attributed to Gerolamo Cardano (1501-1576), whose book Arts Magna (The Great Art) contains the solution of the cubic equation. In fact, it was his contemporary, Bologna's mathematician Rafael Bombelli (1526-1572) who first thoroughly understood the complex numbers and described them in his L'Algebra, published in 1572.)

The multiplicativity of the norm of the quaternions was noted by Euler in 1748, a century before Hamilton discovered the algebra of quaternions in 1843 (when "in a famous act of a mathematical vandalism, he carved the equations $\mathrm{i}^{2}=\mathrm{j}^{2}=\mathrm{k}^{2}=$ $\mathrm{ijk}=-1$ into the stone of Brougham Bridge" [3, p. 145]). The corresponding relation for the octonions was discovered by the Danish mathematician Degen in 1818 - again before the discovery of the octonions (in late 1843 - in a letter to Hamilton by his college friend J. Graves). The first publication about octonions appears as an appendix to an otherwise erroneous paper of the English mathematician (at the time, lawyer) Arthur Cayley (1821-1895) in 1845 (see Introduction of [3] and references 17 and 18 therein )

The American algebraist and author of the three volumes History of the Theory of Numbers, Leonard E. Dickson (1874-1954) contributed to the construction of composition algebras in 1919 [10]. The theorem that the only normed division algebras are $\mathbb{R}, \mathbb{C}, \mathbb{H}$ and $\mathbb{O}$ was proven by A. Hurwitz (1859-1919) in 1898 . The extension of this result to alternative (including split) algebras belongs to M. Zorn (1906-1993) in 1930 and 1933. The fact that the only division algebras (without extra structure) have dimensions 1, 2, 4, 8 was established as late as in 1958 (independently by R. Bott and J. Milnor and by M. Kervaire). 


\section{Octonions. Isometries and Automorphisms}

\subsection{Eight Dimensional Alternative Algebras}

The multiplication table of the imaginary octonions (see Application A) can be introduced by first selecting a quaternion subalgebra

$$
e_{j} e_{k}=\epsilon_{j k l} e_{l}-\delta_{j k}, \quad j, k, l=1,2,4, \quad \epsilon_{124}=1=\epsilon_{412}=\epsilon_{241}=1=-\epsilon_{214} .
$$

The somewhat exotic labeling of the units (jumping over 3 ) is justified by the following memorable multiplication rules $\bmod 7$

$$
\begin{array}{r}
e_{i} e_{j}=e_{k} \Rightarrow e_{i+1} e_{j+1}=e_{k+1}, \quad e_{2 i} e_{2 j}=e_{2 k} \equiv e_{2 k(\bmod 7)} \\
e_{7} e_{j}=e_{3 j(\bmod 7)} \quad \text { for } \quad j=1,2,4, \quad e_{7} e_{4}=e_{5} .
\end{array}
$$

A convenient complex isotropic basis for the vector representation of the isometry Lie algebra $\mathfrak{s o}(8)$ of $\mathbb{O}$ (which contains the automorphism algebra $\mathfrak{g}_{2}$ of the octonions) is given by

$$
\rho^{\epsilon}=\frac{1}{2}\left(1+\mathrm{i} \epsilon e_{7}\right), \quad \zeta_{j}^{\epsilon}=\rho^{\epsilon} e_{j}=\frac{1}{2}\left(e_{j}+\mathrm{i} \epsilon e_{3 j}\right), \quad j=1,2,4, \quad \epsilon= \pm
$$

(the imaginary unit i commutes with octonion units $e_{a}$ ). The multiplication table of the octonion units is summarized by the following relations

$$
\begin{gathered}
\left(\zeta_{j}^{\epsilon}\right)^{2}=0=\rho^{+} \rho^{-}, \quad\left(\rho^{\epsilon}\right)^{2}=\rho^{\epsilon}, \quad \rho^{+}+\rho^{-}=1, \quad \zeta_{j}^{\epsilon} \zeta_{k}^{\epsilon}=\epsilon_{j k l} \zeta_{l}^{-\epsilon} \\
\zeta_{j}^{\epsilon} \zeta_{k}^{-\epsilon}=-\rho^{-\epsilon} \delta_{j k} \quad \Rightarrow \quad\left[\zeta_{j}^{+}, \zeta_{k}^{-}\right]_{+}=\delta_{j k}, \quad j, k, l=1,2,4 .
\end{gathered}
$$

The idempotents $\rho^{ \pm}$(which go back to Gürsey) are also exploited in [12]. The last equation (30) coincides with the canonical anticommutation relations for fermionic creation and annihilation operators (cf. [8]).

The split octonions $x_{s}$ with units $\tilde{e}_{a}$ can be embedded in the algebra $\mathbb{C} \mathbb{O}$ of complexified octonions by setting $\tilde{e}_{\mu}=e_{\mu}, \mu=0,1,2,4, \tilde{e}_{7}=\mathrm{i} e_{7}, \tilde{e}_{3 j}=\mathrm{i} e_{3 j(\bmod 7)}$, so that

$$
\begin{aligned}
x_{s} & =\sum_{a=0}^{7} x_{s}^{a} \tilde{e}_{a} \Rightarrow N\left(x_{s}\right)=x_{s} x_{s}^{*} \\
& =\sum_{\mu=0,1,2,4}\left(x_{s}^{\mu}\right)^{2}-\left(x_{s}^{7}\right)^{2}-\left(x_{s}^{3}\right)^{2}-\left(x_{s}^{6}\right)^{2}-\left(x_{s}^{5}\right)^{2} .
\end{aligned}
$$

The quark-lepton correspondence suggests the splitting of octonions into a direct sum,

$$
\begin{aligned}
& \mathbb{O}=\mathbb{C} \oplus \mathbb{C}^{3}, \quad x=a+\mathbf{z} \mathbf{e}=a+z^{1} e_{1}+z^{2} e_{2}+z^{4} e_{4}, \quad e_{1} e_{2}=e_{4} \\
& a=x^{0}+x^{7} e_{7}, \quad z^{j}=x^{j}+x^{3 j(\bmod 7)} e_{7}, \quad x^{12} \equiv x^{5}
\end{aligned}
$$


thus $e_{7}$ playing the role of imaginary unit within the real octonions. The CayleyDickson construction corresponds to the splitting of $\mathbb{O}$ into two quaternions

$$
\mathbb{O}=\mathbb{H} \oplus \mathbb{H}, \quad x=u+e_{7} v, \quad u=x^{0}+x^{j} e_{j}, \quad v=x^{7}+x^{3 j} e_{j} .
$$

One may speculate that upon complexification $u$ and $v$ could be identified with the "up-" and "down-", leptons and quarks: $u=\left(\nu, u^{j}, j=1,2,4\right) . v=\left(e^{-}, d_{j}\right)$ $j$ playing the role of a colour index. For $x_{r}=u_{r}+e_{7} v_{r}, r=1,2$ the CayleyDickson formula (16) and its expression in terms of the complex variable $a_{r}, z_{r}^{j}$ read

$$
\begin{aligned}
x_{1} x_{2}=u_{1} u_{2}-v_{2} v_{1}^{*}+e_{7} & \left(u_{1}^{*} v_{2}+u_{2} v_{1}\right) \\
& =a_{1} a_{2}-\mathbf{z}_{\mathbf{1}} \overline{\mathbf{z}}+\left(a_{1} \mathbf{z}_{2}+\bar{a}_{2} \mathbf{z}_{\mathbf{1}}+\overline{\mathbf{z}}_{\mathbf{1}} \times \overline{\mathbf{z}}_{\mathbf{2}}\right) \mathbf{e}
\end{aligned}
$$

where the star indicates quaternionic conjugation while the bar stands for a change of the sign of $e_{7}\left(\bar{z}^{j}=x^{j}-e_{7} x^{3 j}\right)$. The two representations (34) display the covariance of the product under the action of two subgroups of maximal rank of the automorphism group of the octonions. If $p$ and $q$ are two unit quaternions

$$
\begin{aligned}
p & =p^{0}+p^{j} e_{j}, \quad q=q^{0}+q^{j} e_{j} \\
p p^{*} & =N(p)=\left(p^{0}\right)^{2}+\mathbf{p}^{2}=1=q q^{*} \Leftrightarrow(p, q) \in \mathrm{SU}(2) \times \mathrm{SU}(2) .
\end{aligned}
$$

It is easy to verify, using the first equation (34), that the transformation

$$
\begin{aligned}
x & =u+e_{7} v \rightarrow \quad \rightarrow \quad p u p^{*}+e_{7} p v q^{*} \\
(p, q) & \in \frac{\mathrm{SU}(2) \times \mathrm{SU}(2)}{\mathbb{Z}_{2}^{\text {diag }}}
\end{aligned}
$$

where $\mathbb{Z}_{2}^{\text {diag }}=\{(p, q)=(1,1),(-1,-1)\}$, is an automorphism of the octonion algebra. Similarly, if $U \in \mathrm{SU}(3)$ acts on $x$ (32) as

$$
x=a+z^{j} e_{j} \rightarrow a+U_{j}^{i} z^{j} e_{i}, \quad U\left(x_{1}\right) U\left(x_{2}\right)=U\left(x_{1} x_{2}\right) .
$$

The subgroups (36), (37) are the two closed connected subgroups of maximal rank of the compact group $\mathrm{G}_{2}$ corresponding to the Borel-de Siebenthal theory [7] that plays a central role in [30].

\subsection{Isometry Group of the (Split) Octonions. Triality}

The norm $N(x)=\sum_{0}^{7}\left(x^{a}\right)^{2}$ and the associated scalar product are preserved by the orthogonal group $\mathrm{O}(8)$ in 8-dimensions. Similarly, the isometry group of the norm of the split octonions $\tilde{x} \in \mathbb{O}_{s}$

$$
N_{s}(\tilde{x})=\left(\tilde{x}^{0}\right)^{2}+\left(\tilde{x}^{1}\right)^{2}+\left(\tilde{x}^{2}\right)^{2}+\left(\tilde{x}^{4}\right)^{2}-\left(\tilde{x}^{7}\right)^{2}-\left(\tilde{x}^{3}\right)^{2}-\left(\tilde{x}^{6}\right)^{2}-\left(\tilde{x}^{5}\right)^{2}
$$


is the noncompact pseudoorthogonal group $\mathrm{O}(4,4)$. What is remarkable is that the trilinear form $t(x y z)$ (10) is invariant under the 2-fold cover $\operatorname{Spin}(8)$ (respectively, $\operatorname{Spin}(4,4)$ ) of the connected group $\mathrm{SO}(8)$ (respectively, $\mathrm{SO}(4,4)$ ). More precisely, there exist two involutive outer automorphisms ${ }^{3} \kappa$ and $\pi$ of $\operatorname{Spin}(8)$ such that for each element $g \in \operatorname{Spin}(8)$ the trilinear form

$$
t_{8}(x, y, z)=\frac{1}{2} t(x y z)=\langle x y z, 1\rangle
$$

is invariant under the combined transformation $x \rightarrow g x, y \rightarrow \kappa(g) y, z \rightarrow \pi(g) z$

$$
t_{8}(g x, \kappa(g) y, \pi(g) z)=t_{8}(x, y, z) .
$$

The factor $\frac{1}{2}$ in (39) follows from the definition of normed triality of [3] which demands $\left|t_{8}(x, y, z)\right|^{2} \leqq N(x) N(y) N(z)$. We shall illustrate the meaning of $\kappa$ and $\pi$ in the special case when $g$ acts on $x$ by left multiplication with a normone octonion $a: L_{a} x=a x$. Note that not all $\operatorname{Spin}(8)$ elements can be written in this form. For instance, due to non-associativity, the product $L_{a_{1}} L_{a_{2}} x=a_{1}\left(a_{2} x\right)$ cannot, in general be written in the form $L_{a} x$. As emphasized in the thesis [18], the composition of maps $L_{a_{1}} L_{a_{2}}$ is associative while the product of octonions is not. We define, in general, the map $\kappa: \operatorname{Spin}(8) \rightarrow \operatorname{Spin}(8)$ by $\kappa(g) x:=\left(g x^{*}\right)^{*}$ $(x \in \mathbb{O}, g=\operatorname{Spin}(8))$. It ia easy to verify that

$$
\kappa L_{a}=R_{a}, \quad \text { where } \quad R_{a} y=y a^{*}, \quad a, y \in \mathbb{O} ; \quad a a^{*}=1 .
$$

(Note that if the algebra $\mathcal{A}$ of elements $a$ were associative then the group law $R_{a_{1}} R_{a_{2}}=R_{a_{1} a_{2}}$ would be only satisfied if one uses the conjugation for right action as in (41).) Finally, setting

$$
\pi L_{a}=T_{a}, \quad \text { where } \quad T_{a} z=a z a^{*}, \quad \pi R_{a}=R_{a}, \quad \pi T_{a}=L_{a}
$$

we verify the invariance condition (40). The case of a general $g$ can be deduced from here by first proving that any element of $g$ can be represented as a product of a finite number of left multiplications (cf. [11,32]).

\subsection{Automorphism Group and Derivations of Octonions}

As proven by Elie Cartan in 1914, the automorphism group of the octonions is the exceptional Lie group

$$
\mathrm{G}_{2}=\{\mathrm{g} \in \mathrm{L}(\mathbb{O}, \mathbb{R}) ;(\mathrm{gx})(\mathrm{gy})=\mathrm{g}(\mathrm{xy}), \quad \mathrm{x}, \mathrm{y} \in \mathbb{O}\}
$$

\footnotetext{
${ }^{3}$ We adopt the notation of [32] where $\kappa, \pi$ and $\nu=\kappa \pi$ are defined as Lie algebra automorphisms. Baez ([3] Section 2.4), who works with the group action, denotes them $\alpha^{ \pm}$.
} 
where $\mathrm{L}(\mathbb{O}, \mathbb{R})$ is the group of non-singular linear transformations of the 8-dimensional real vector space $\mathbb{O}$. It follows from (38) that $\mathrm{G}_{2}$ preserves the octonion unit and commutes with the Cayley conjugation, so that it preserves the norm $N(x)$

$$
g 1=1, \quad(g x)^{*}=g\left(x^{*}\right), \quad N(g x)=N(x) .
$$

Thus $\mathrm{G}_{2}$ is a subgroup of the isometry (orthogonal) group $\mathrm{O}(\mathbb{O})=\mathrm{O}(8)$ of the 8 -dimensional euclidean space of the octonions. In fact, it is a subgroup of the connected orthogonal group $\mathrm{SO}(7)$ of the 7-dimensional space $\mathbb{O}_{0}$ of imaginary octonions, the Lie algebra $\mathfrak{s o}(7)$ splitting as a vector space into a direct sum of the Lie algebra $\mathfrak{g}_{2}$ of $\mathrm{G}_{2}$ and its lowest order faithful 7-dimensional representation

$$
\mathfrak{s o}(7) \approx \mathfrak{g}_{2} \oplus \underline{7} \cong \mathfrak{g}_{2} \oplus \mathbb{R}^{7} .
$$

We see, in particular, that the dimension of $\mathrm{G}_{2}$ is 14 .

The maximal subgroups of $\mathrm{G}_{2}$, whose action was defined by (36) and (37) (and which correspond to the Borel-de Siebenthal theory) can be characterized as follows. The complex conjugation $\gamma$ (in the notation of [32]): $e_{7} \rightarrow-e_{7}$ belongs to the automorphism group $\mathrm{G}_{2}$ of $\mathbb{O}$ (corresponding, in fact, to the reflection of four imaginary units $e_{7}, e_{7} e_{1}=e_{3}, e_{7} e_{2}=e_{6}$ and $\left.e_{7} e_{4}=e_{5}\right)$ and has square one

$$
\gamma x=\gamma\left(u+e_{7} v\right)=u-e_{7} v, \quad \gamma^{2}=1 .
$$

The rank two (semisimple) subgroup (35), (36) of $\mathrm{G}_{2}$ can be characterized as the commutant of $\gamma$ in $\mathrm{G}_{2}$

$$
\mathrm{G}_{2}^{\gamma}=\left\{\mathrm{g} \in \mathrm{G}_{2} ; \gamma \mathrm{g}=\mathrm{g} \gamma\right\} \equiv \frac{\mathrm{SU}(2) \times \mathrm{SU}(2)}{\mathbb{Z}_{2}} .
$$

Denote, on the other hand, by $\omega$ the generator of the center of $\mathrm{SU}(3)$ acting on $z$ by (37)

$$
\omega x=a+\omega_{7} z^{j} e_{j}, \quad \omega_{7}=-\frac{1}{2}+\frac{\sqrt{3}}{2} e_{7}, \quad \omega_{7}^{3}=1=\omega^{3} .
$$

Then the subgroup (37) of $G_{2}$ is characterized by

$$
\mathrm{G}_{2}^{\omega}:=\left\{\omega \in \mathrm{G}_{2} ; \omega \mathrm{g}=\mathrm{g} \omega\right\} \equiv \mathrm{SU}(3) .
$$

It is convenient to embed the Lie algebra $\mathfrak{s o}(7)$ (and hence $\mathfrak{g}_{2} \subset \mathfrak{s o}(7)$ ) into the isometry algebra $\mathfrak{s o}(8)$ of the quadratic form $N(x)$. Following Yokota [32] we introduce two bases ${ }^{4} G_{a b}\left(=G_{b a}\right)$ and $F_{a b}$ in the 28-dimensional vector space

\footnotetext{
${ }^{4}$ We note that the convention $e_{1} e_{2}=e_{3}$ (rather then our $e_{1} e_{2}=e_{4}$ ) is used in [32] so that the relations connecting $G$ and $F$ (Application B) are different.
} 
$\mathfrak{s o}(8)$

$$
\begin{aligned}
G_{a b} e_{c} & =\delta_{b c} e_{a}-\delta_{a c} e_{b}, \quad a, b, c=0,1, \ldots, 7, \quad e_{0}=1 \\
F_{a b} x & =\frac{1}{2} e_{a}\left(e_{b}^{*} x\right)=-F_{b a} x, \quad F_{a a} \equiv 0, \quad e_{0}^{*}=e_{0}
\end{aligned}
$$

related by an involution $\pi$

$$
(\pi G)_{a b}=F_{a b}, \quad(\pi F)_{a b}=G_{a b}, \quad \pi^{2}=1 .
$$

We shall demonstrate in Application B that the involution $\pi$ splits into seven fourdimensional involutive transformations. Here, we display one of them which involves our choice of the Cartan subalgebra of $\mathfrak{s o}(8)$

$$
F_{7}=X_{7} G_{7}, G_{7}=\left(\begin{array}{l}
G_{07} \\
G_{13} \\
G_{26} \\
G_{45}
\end{array}\right), F_{7}=\left(\begin{array}{l}
F_{07} \\
F_{13} \\
F_{26} \\
F_{45}
\end{array}\right), X_{1}=\frac{1}{2}\left(\begin{array}{rrrr}
1 & 1 & 1 & 1 \\
1 & 1 & -1 & -1 \\
1 & -1 & 1 & -1 \\
1 & -1 & -1 & 1
\end{array}\right)
$$

a straightforward calculation gives $X_{7}^{2}=\mathbb{1}$, $\operatorname{det} X_{7}=-1$. The generators $G_{a b}$ obey the following commutation relations

$$
\left[G_{a b}, G_{c d}\right]=2\left(\delta_{b c} G_{a d}-\delta_{a c} G_{b d}+\delta_{a d} G_{b c}-\delta_{b d} G_{a c}\right) .
$$

In order to work with real Cartan matrices we shall first display the isometry algebra $\mathfrak{s o}(4,4)$ and the derivations of the split octonions defined by (31). The algebra $\mathfrak{s o}(4,4)$ has 12 compact and 16 noncompact generators. The maximal compact subalgebra $\mathfrak{s o}(4) \times \mathfrak{s o}(4)$ of ${ }^{5} D_{4(4)}=\mathfrak{s o}(4,4)$ is spanned by the generators $G_{\mu \nu}$, $\mu, \nu=0,1,2,4,\left(\mu\langle\nu) ; G_{3 j, 7}, G_{3 j, 3 k}, j, k=1,2,4, j\langle k\right.$ while the 16 noncompact generators are given by

$$
\begin{aligned}
\tilde{G}_{\mu, 3 \rho} & =\frac{1}{\mathrm{i}} G_{\mu, 3 \rho}=\tilde{G}_{3 \rho, \mu}, \quad \mu=0,1,2,4, \quad 3 \rho=3,6,5,7 \\
3 \rho & =3 \rho(\bmod 7), \quad \text { so that } \quad 3 \times 4=5,3 \times 7=7 .
\end{aligned}
$$

\subsection{Roots and Weights of $\mathfrak{g}_{2(2)} \subset \mathfrak{s o}(4,4)$}

The Lie algebra $\mathfrak{s o}(8)$ being simply laced, the roots $\alpha_{j}$ coincide with coroots $\alpha_{j}^{\vee}=2 \alpha_{j} / \alpha_{j}^{2}$. The four-dimensional root space is spanned by the orthogonal weight basis $\left\{\lambda_{\mu}\right\}$

$$
\lambda_{0} \leftrightarrow H_{0}=\tilde{G}_{07}, \lambda_{1} \leftrightarrow H_{1}=\tilde{G}_{13}, \lambda_{2} \leftrightarrow H_{2}=\tilde{G}_{26}, \lambda_{4} \leftrightarrow H_{4}=\tilde{G}_{45} .
$$

\footnotetext{
${ }^{5}$ The notation $\mathrm{L}_{\mathrm{r}(\mathrm{s})}$ for a rank $r$ Lie algebra $\mathrm{L}_{\mathrm{r}}$ means that its signature, the difference between the number of + and - signs of its Cartan Killing form, is $s$.
} 
In the isotropic basis (29) $H_{\mu}$ are $8 \times 8$ diagonal matrices with two non-zero eigenvalues

$$
H_{\mu} \zeta_{\nu}^{\epsilon}=\epsilon \delta_{\mu \nu} \zeta_{\nu}^{\epsilon}, \quad \epsilon= \pm, \quad \mu, \nu=0,1,2,4
$$

so that we have

$$
\left\langle\lambda_{\mu}, \lambda_{\nu}\right\rangle=\frac{1}{2} \operatorname{tr} H_{\mu} H_{\nu}=\delta_{\mu \nu}, \quad \zeta_{0}^{\epsilon}=\rho^{\epsilon} .
$$

The simple roots $\alpha_{\nu}$ and the fundamental weights $\lambda_{\mu}$ of $\mathfrak{s o}(4,4)$ are

$$
\begin{gathered}
\alpha_{0}=\lambda_{0}-\lambda_{1} \leftrightarrow H_{01}=H_{0}-H_{1}, \quad \alpha_{1}=\lambda_{1}-\lambda_{2} \leftrightarrow H_{12}=H_{1}-H_{2} \\
\alpha_{2}^{-}=\lambda_{2}-\lambda_{3} \leftrightarrow H_{2}-H_{3} \alpha_{2}^{+}=\lambda_{2}+\lambda_{3} \leftrightarrow H_{2}+H_{3} \\
\Lambda_{0}=\lambda_{0}, \quad \Lambda_{1}=\lambda_{0}+\lambda_{1}, \quad \Lambda_{2}^{\mp}=\frac{1}{2}\left(\lambda_{0}+\lambda_{1}+\lambda_{2} \mp \lambda_{3}\right) \\
\quad\left\langle\Lambda_{\mu}^{\epsilon}, \alpha_{\nu}^{\epsilon^{\prime}}\right\rangle=\delta_{\mu \nu} \delta^{\epsilon \epsilon^{\prime}}, \quad \epsilon= \pm, \quad \mu, \nu=0,1,2 .
\end{gathered}
$$

The rank three- Lie subalgebra $\mathfrak{s o}(7) \subset \mathfrak{s o}(8)$ is spanned by the 21 generators $G_{k l}$ with $k, l>0$. The simple (co)roots and the fundamental weights of the noncompact real form $\mathfrak{s o}(4,3)$ and the corresponding Cartan matrix of $\mathfrak{s o}(7, \mathbb{C})$ are

$$
\begin{gathered}
\alpha_{1}=\lambda_{1}-\lambda_{2}, \quad \alpha_{2}^{-}=\lambda_{2}-\lambda_{3}, \quad \alpha_{3}=\lambda_{3} \Rightarrow \alpha_{3}^{\vee}=2 \alpha_{3} \\
\Lambda_{1}^{\mathfrak{s o}(7)}=\lambda_{1}, \quad \Lambda_{2}^{\mathfrak{s o}(7)}=\lambda_{1}+\lambda_{2}, \quad \Lambda_{3}^{\mathfrak{s o}(7)}=\frac{1}{2}\left(\lambda_{1}+\lambda_{2}+\lambda_{3}\right) \\
\left\langle\Lambda_{j}^{\mathfrak{s o}(7)}, \alpha_{k}^{\vee}\right\rangle=\delta_{j k}, \quad\left(c_{j k}\right)=\left(\left\langle\alpha_{j}^{\vee}, \alpha_{k}\right\rangle\right)=\left(\begin{array}{rrr}
2-1 & 0 \\
-1 & 2 & -1 \\
0 & -2 & 2
\end{array}\right) .
\end{gathered}
$$

Finally, the derivation algebra $\mathfrak{g}_{2}(\subset \mathfrak{s o}(7)$ of the octonions is spanned by

$$
\begin{array}{ll}
\lambda G_{24}+\mu G_{37}+\nu G_{56}, & \lambda G_{14}-\mu G_{35}+\nu G_{76} \\
\lambda G_{17}+\mu G_{25}-\nu G_{46}, & -\lambda G_{12}+\mu G_{36}+\nu G_{75} \\
\lambda G_{16}-\mu G_{23}+\nu G_{47}, & -\lambda G_{15}+\mu G_{27}+\nu G_{43} \\
\lambda G_{13}+\mu G_{26}+\nu G_{45}, & \lambda+\mu+\nu=0 .
\end{array}
$$

For instance, the last line of (63) is a symmetric way of saying that the Cartan subalgebra of $\mathfrak{g}_{2}$ is spanned by $G_{13}-G_{26}, G_{26}-G_{45}$. Thus $\mathfrak{g}_{2}$ is $7 \times 2=14$ dimensional. Its simple (co-)roots are expressed conveniently in terms of the barycentric weights

$$
\bar{\lambda}_{j}:=\lambda_{j}-\frac{1}{3}\left(\lambda_{1}+\lambda_{2}+\lambda_{4}\right), \quad j=1,2,4, \quad \sum_{j} \bar{\lambda}_{j}=0
$$


as follows

$$
\begin{aligned}
& \alpha_{1}=\bar{\lambda}_{1}-\bar{\lambda}_{2} \quad\left(=\lambda_{1}-\lambda_{2}\right) \quad \longleftrightarrow \quad H_{12}=\tilde{G}_{13}-\tilde{G}_{26} \\
& \alpha_{2}=\bar{\lambda}_{2} \quad \Rightarrow \quad \alpha_{2}^{\vee}=3 \alpha_{2}
\end{aligned}
$$

yielding the $\mathfrak{g}_{2}$ Cartan matrix

$$
\left(\left\langle\alpha_{i}^{\vee}, \alpha_{j}\right\rangle\right)=\left(\begin{array}{rr}
2 & -1 \\
-3 & 2
\end{array}\right)
$$

The remaining four positive roots of $\mathfrak{g}_{2}$ (including the fundamental weights $\Lambda_{i}^{\mathfrak{g}_{2}}$ ) are given by

$$
\begin{aligned}
\alpha_{1}+\alpha_{2} & =\bar{\lambda}_{1}, \quad \alpha_{1}+2 \alpha_{2}=\bar{\lambda}_{1}+\bar{\lambda}_{2}=-\bar{\lambda}_{4}=\Lambda_{2}^{\mathfrak{g}_{2}} \\
\alpha_{1}+3 \alpha_{2} & =\bar{\lambda}_{2} \bar{\lambda}_{4}=\lambda_{2}-\lambda_{4} \quad \longleftrightarrow \quad H_{24}=\tilde{G}_{26}-\tilde{G}_{45} \\
\theta: & =2 \alpha_{1}+3 \alpha_{2}=\bar{\lambda}_{1}-\bar{\lambda}_{4} \\
& =\lambda_{1}-\lambda_{4}=\Lambda_{1}^{\mathfrak{g}_{2}} \longleftrightarrow \quad H_{14}=\tilde{G}_{13}-\tilde{G}_{45} .
\end{aligned}
$$

The $\mathfrak{s u}(3)$ subalgebra of $\mathfrak{g}_{2}$ becomes $\mathfrak{s l}_{3}=\mathfrak{s l}(3, \mathbb{R})$ in $\mathfrak{g}_{2(2)}$. Its Cartan matrices are $H_{12}, H_{24}$ while the raising (and the lowering) generators $E_{\alpha}$ (and $F_{\alpha}$ ) are labeled by the long roots $\alpha=\alpha_{1}, \alpha_{1}+3 \alpha_{2}, \theta$. The maximal compact subalgebra $\mathfrak{s o}(3)$ of $\mathfrak{s l}_{3}$ is spanned by

$$
\begin{aligned}
L_{12} & :=G_{12}+G_{36}, \quad L_{24}=G_{24}+G_{56}, \quad L_{14}=G_{14}+G_{35} \\
{\left[L_{12}, L_{24}\right] } & =L_{14}, \quad\left[L_{12}, L_{14}\right]=-L_{24}=L_{42}
\end{aligned}
$$

the non-diagonal non-compact generators accompanying (64) are

$$
\tilde{G}_{16}+\tilde{G}_{23}, \quad \tilde{G}_{15}+\tilde{G}_{13}, \quad \tilde{G}_{14}+\tilde{G}_{35} .
$$

\section{Jordan Algebras and Related Groups}

\subsection{Classification of Finite Dimensional Jordan Algebras}

Pascual Jordan (1902-1980) the "unsung hero among the creators of quantum theory" (in the words of Schweber, 1994) asked himself in 1932 a question you would expect of an idle mathematician: Can one construct an algebra of (hermitian) observables without introducing an auxiliary associative product? He arrived, after some experimenting with the special Jordan product

$$
A \circ B=\frac{1}{2}(A B+B A)=B \circ A
$$


at two axioms (Jordan, 1933)

$$
\text { i) } A \circ B=B \circ A, \quad \text { ii) } A^{2} \circ(B \circ A)=\left(A^{2} \circ B\right) \circ A
$$

where $A^{2}:=(A \circ A)$. They imply, in particular, power associativity and

$$
A^{m} \circ A^{n}=A^{m+n}, \quad m, n=0,1,2, \ldots, \quad A^{0}=1 .
$$

Being interested in extracting the properties of the algebra of hermitian matrices (or self-adjoint operators) for which $A^{2} \geq 0$, Jordan adopted Artin's formal reality condition

$$
A_{1}^{2}+\cdots+A_{n}^{2}=0 \quad \Longrightarrow \quad A_{1}=0=\cdots=A_{n} .
$$

In a fundamental paper of 1934 Jordan, von Neumann and Wigner [23] classified all finite dimensional formally real Jordan algebras (i.e., algebras over the field of real numbers satisfying (67). They split into a direct sum of simple algebras, which belong to four infinite families

$$
\mathcal{H}_{n}(\mathbb{R}), \mathcal{H}_{n}(\mathbb{C}), \mathcal{H}_{n}(\mathbb{H}), \operatorname{JSpin}(n)
$$

and a single exceptional one

$$
\mathfrak{J}\left(=\mathfrak{J}_{3}^{8}\right)=\mathcal{H}_{3}(\mathbb{O}) .
$$

Here $\mathcal{H}_{n}(\mathbb{A})$ stands for the set of $n \times n$ hermitian matrices with entry in the division ring $\mathbb{A}(=\mathbb{R}, \mathbb{C}, \mathbb{H}, \mathbb{O})$, equipped with the commutative product (66). (One uses the same notation when $\mathbb{A}$ is replaced by one of the alternative split composition rings, $\mathbb{C}_{s}, \mathbb{H}_{s}$ or $\mathbb{O}_{s}$ albeit the resulting algebra is not formally real in that case.) JSpin $(n)$ is an algebra of elements $\left(\xi, x ; \xi \in \mathbb{R}, x \in \mathbb{R}^{n}\right)$ where $\mathbb{R}^{n}$ is equipped with the (real) euclidean scalar product $\langle x, y\rangle$ and the product in $\operatorname{JSpin}(n)$ is given by

$$
(\xi, x)(\eta, y)=(\xi \eta+\langle x, y\rangle, \xi y+\eta x) .
$$

The first three algebras $\mathcal{H}_{n}(\mathbb{A})(70)$ are equipped with the special Jordan product (66) where $A B$ stands for the (associative) matrix product. The algebra $\operatorname{JSpin}(n)$ is special as a Jordan subalgebra of the $2^{n}$ dimensional (associative) Clifford algebra $\operatorname{Cliff}(\mathrm{n})$.

Remark 4. The Jordan algebras $\mathcal{H}_{2}(\mathbb{A})$ for $\mathbb{A}=\mathbb{R}, \mathbb{C}, \mathbb{H}, \mathbb{O}$ are isomorphic to $\operatorname{JSpin}(n)$ for $n=2,3,5,9$ respectively. Indeed in all four cases the elements of $\mathbb{A}$ split into a multiple of the unit matrix and a traceless part of scalar square

$$
\begin{gathered}
X=\left(\begin{array}{cc}
\xi_{1} & x \\
x^{*} \xi_{2}
\end{array}\right)=\xi_{0} l+X_{0} \xi_{0}=\frac{\xi_{1}+\xi_{2}}{2}, \quad X_{0}=\left(\begin{array}{cc}
\xi & x \\
x^{*}-\xi
\end{array}\right) \\
\xi=\frac{\xi_{1}-\xi_{2}}{2}, \quad X_{0}^{2}=\left(\xi^{2}+x x^{*}\right) l, \quad x x^{*}=x^{*} x \in \mathbb{R}_{+} .
\end{gathered}
$$


We note that in each case the determinant of $X$ has a Minkovski space signature

$$
\operatorname{det} X=\xi_{0}^{2}-\xi^{2}-x x^{*}=\xi_{1} \xi_{2}-x x^{*}
$$

and is thus invariant under the Lorentz group in 3, 4, 6 and 10 dimensions, respectively.

On the other hand, the algebras $\mathcal{H}_{n}(\mathbb{O})$ for $n>3$ are not Jordan since they violate condition ii) of (67). The exceptional Jordan algebra $\mathfrak{J}=\mathcal{H}_{3}(\mathbb{O})$ did not seem to be special but the authors of [23] assigned the proof that the product $A \circ B$ of two elements of $\mathfrak{J}$ cannot be represented in the form (66) with an associative product. The PhD student of L. Dickson Abraham Albert (1905-1972) proved this.

We introduce the one-dimensional projectors $E_{i}$ and the hermitian octonionic matrices $F_{i}\left(x_{i}\right)$ writing down a general element of $\mathcal{H}_{3}(\mathbb{O})$ as

$$
X=\left(\begin{array}{ccc}
\xi_{1} & x_{3} & x_{2}^{*} \\
x_{3}^{*} & \xi_{2} & x_{1} \\
x_{2} & x_{1}^{*} & \xi_{3}
\end{array}\right)=\xi_{1} E_{1}+\xi_{2} E_{2}+\xi_{3} E_{3}+F_{1}\left(x_{1}\right)+F_{2}\left(x_{2}\right)+F_{3}\left(x_{3}\right) .
$$

We can then write the Jordan multiplication $X \circ Y$ setting

$$
\begin{gathered}
E_{i} \circ E_{j}=\delta_{i j} E_{j}, \quad E_{i} \circ F_{j}(x)= \begin{cases}0, & \text { if } \quad i=j \\
\frac{1}{2} F_{j}(x), & \text { if } \quad i \neq j\end{cases} \\
F_{i}(x) \circ F_{i}(y)=\langle x, y\rangle\left(E_{i+1}+E_{i+2}\right), \quad F_{i}(x) F_{i+1}(y)=\frac{1}{2} F_{i+2}\left(y^{*} x^{*}\right)
\end{gathered}
$$

where the indices are counted $\bmod 3: E_{4} \equiv E_{1}, F_{5} \equiv F_{2}, \ldots$ We define the trace, a symmetric bilinear inner product and a trilinear scalar product in $\mathfrak{J}$ by

$$
\operatorname{tr} X=\xi_{1}+\xi_{2}+\xi_{3}, \quad\langle X, Y\rangle=\operatorname{tr}(X \circ Y), \quad \operatorname{tr}(X, Y, Z)=\langle X, Y \circ Z\rangle .
$$

The Jordan algebra $\mathfrak{J}$ also admits a (symmetric) Freudenthal product

$$
X \times Y=\frac{1}{2}[2 X \circ Y-X \operatorname{tr} Y-Y \operatorname{tr} X+(\operatorname{tr} X \operatorname{tr} Y-\langle X, Y\rangle E)]
$$

where $E$ is the $3 \times 3$ unit matrix, $E=E_{1}+E_{2}+E_{3}$. Finally, we define a three-linear form $(X, Y, Z)$ and the determinant $\operatorname{det} X$ by

$$
\begin{aligned}
(X, Y, Z) & =\langle X, Y \times Z\rangle=\langle X \times Y, Z\rangle, \quad \operatorname{det} X=\frac{1}{3}(X, X, X) \\
& =\xi_{1} \xi_{2} \xi_{3}+2 \operatorname{Re}\left(x_{1} x_{2} x_{3}\right)-\xi_{1} x_{1} x_{1}^{*}-\xi_{2} x_{2} x_{2}^{*}-\xi_{3} x_{3} x_{3}^{*} .
\end{aligned}
$$

The following identities hold

$$
X \times X \circ X=(\operatorname{det} X) E, \quad(X \times X) \times(X \times X)=(\operatorname{det} X) X .
$$




\subsection{Automorphism Groups of the Exceptional Jordan Algebras $\mathcal{H}_{3}\left(\mathbb{O}_{(s)}\right)$ and their Maximal Subgroups}

Classical Lie groups appear as symmetries of classical symmetric spaces. For quite some time there was no such interpretation for the exceptional Lie groups. The situation only changed with the discovery of the exceptional Jordan algebra $\mathcal{H}_{3}(\mathbb{O})$ and its split octonions' cousin $\mathcal{H}_{3}\left(\mathbb{O}_{s}\right)$.

The automorphism group of the $\mathcal{H}_{3}(\mathbb{O})$ algebra is the rank four compact simple Lie group ${ }^{6} \mathrm{~F}_{4}$. It clearly leaves the unit element $E$ invariant and is proven to preserve the trace (77) (see Lemma 2.2.1 in [32]). The stabilizer of $E_{1}$ in $\mathrm{F}_{4}$ is the double covering $\operatorname{Spin}(9)$ of the rotation group in nine dimensions (which preserves $\left.X_{0}^{2}(73)\right)$. Moreover, we have

$$
\mathrm{F}_{4} / \operatorname{Spin}(9) \simeq \mathbb{O P}^{2} \Longrightarrow \operatorname{dim} \mathrm{F}_{4}=\operatorname{dim} \operatorname{Spin}(9)+\operatorname{dim} \mathbb{O}^{2}=36+16=52 \text {. }
$$

Building upon our treatment of $\mathfrak{g}_{2(2)}$ (Section 2.2) we shall first construct the Lie algebra $\mathfrak{f}_{4(4)}$ of derivations (infinitesimal automorphisms) of $\mathcal{H}_{3}\left(\mathbb{O}_{s}\right)$ which admits a real Cartan subalgebra spanned by the orthonormal basis $\lambda_{0}, \lambda_{1}, \lambda_{2}, \lambda_{4}$ (55) (normalized by (57)). The simple roots of $\mathfrak{f}_{4(4)}$ (and the associated Cartan matrices in the basis $\left.\zeta_{\mu}^{\epsilon}(29)\right)$ are:

$$
\begin{aligned}
& \alpha_{1}=\lambda_{1}-\lambda_{2} \longleftrightarrow H_{12}=\epsilon\left(\begin{array}{rrrr}
0 & 0 & 0 & 0 \\
0 & 1 & 0 & 0 \\
0 & 0 & -1 & 0 \\
0 & 0 & 0 & 0
\end{array}\right) \\
& \alpha_{2}=\lambda_{2}-\lambda_{4} \longleftrightarrow H_{24}=H_{2}-H_{4} \\
& \alpha_{4}=\lambda_{4} \longleftrightarrow H_{4}=\epsilon\left(\begin{array}{llll}
0 & 0 & 0 & 0 \\
0 & 0 & 0 & 0 \\
0 & 0 & 0 & 0 \\
0 & 0 & 0 & 1
\end{array}\right) \\
& \alpha_{0}=\frac{1}{2}\left(\lambda_{0}-\lambda_{1}-\lambda_{2}-\lambda_{4}\right) \\
& \longleftrightarrow \frac{1}{2}\left(H_{01}-H_{2}-H_{4}\right)=\frac{\epsilon}{2}\left(\begin{array}{rrrr}
1 & 0 & 0 & 0 \\
0 & -1 & 0 & 0 \\
0 & 0 & -1 & 0 \\
0 & 0 & 0 & -1
\end{array}\right) .
\end{aligned}
$$

\footnotetext{
${ }^{6}$ This was proven by Claude Chevalley and Richard Schafer in 1950. The result was prepared by Ruth Moufang's study in 1933 of the octonionic projective plane, then Jordan's construction in 1949 of $\mathbb{O P}^{2}$ in terms of one-dimensional projections in $\mathcal{H}_{3}(\mathbb{O})$ and Armand Borel's observation that $\mathrm{F}_{4}$ is the isometry group of $\mathbb{O P}^{2}$. For a review and references - see [3, Section 4.2]. Octonionic quantum mechanics in the Moufang plane was considered in [20].
} 
The corresponding coroots and Cartan matrix for $\mathfrak{f}_{4}$ are

$$
\begin{array}{r}
\alpha_{1}^{\vee}=\alpha_{1}, \quad \alpha_{2}^{\vee}=\alpha_{2}, \quad \alpha_{4}^{\vee}=2 \alpha_{4}, \quad \alpha_{0}^{\vee}=2 \alpha_{0} \\
\left(c_{i j}=\left\langle\alpha_{i}^{\vee}, \alpha_{j}\right\rangle\right)=\left(\begin{array}{rrrr}
2 & -1 & 0 & 0 \\
-1 & 2 & -1 & 0 \\
0 & -2 & 2 & -1 \\
0 & 0 & -1 & 2
\end{array}\right) .
\end{array}
$$

The Lie algebra $\mathfrak{f}_{4(4)}$ has 24 positive roots: the 12 long roots coincide with the positive roots $\left\{\lambda_{0} \pm \lambda_{j}, j=1,2,4, \lambda_{j} \pm \lambda_{k}, 1 \leqq j\langle k \leqq 4\}\right.$ of $\mathfrak{s o}(4,4)$; the 4 short roots $\lambda_{\mu}, \mu=0,1,2,4$ coincide with the short positive roots of $\mathfrak{s o}(5,4)$; finally, $\mathfrak{f}_{4(4)}$ has 8 additional short roots of the form $\frac{1}{2}\left(\lambda_{0} \pm \lambda_{1}, \pm \lambda_{2} \pm \lambda_{4}\right)$; the highest root $\theta$ of $\mathfrak{f}_{4(4)}$ coincide with that of its rank four simple subalgebras $\mathfrak{s o}(5,4)$ and $\mathfrak{s o}(4,4)$

$$
\theta=\lambda_{0}+\lambda_{1}=2 \alpha_{1}+3 \alpha_{2}+4 \alpha_{4}+2 \alpha_{0} .
$$

The elements $D$ of $\mathfrak{s o}(8)$ act on $X$ of $\mathfrak{J}(75)$ through their action on the octonions.

$$
D X=F_{1}\left(D x_{1}\right)+F_{2}\left(\kappa(D) x_{2}\right)+F_{3}\left(\pi(D) x_{3}\right)
$$

where $D=: D_{1}, \kappa(D)=: D_{2}, \pi(D)=: D_{3}$, obey the principle of infinitesimal triality

$$
\left(D_{1} x\right) y+x\left(D_{2} y\right)=\left(D_{3}\left((x y)^{*}\right)\right)^{*} .
$$

For $D \in \mathrm{G}_{2}$ we have $D_{1}=D_{2}=D_{3}=D$.

The remaining 24 generators of $\mathfrak{f}_{4}$ (outside $\mathfrak{s o}(8)$ ) can be identified with the skewhermitian matrices $A_{i}\left(e_{a}\right), i=1,2,3, a=0,1, \ldots, 7$

$$
A_{1}(x)=\left(\begin{array}{ccc}
0 & 0 & 0 \\
0 & 0 & x \\
0 & -x^{*} & 0
\end{array}\right), A_{2}(x)=\left(\begin{array}{ccc}
0 & 0 & -x^{*} \\
0 & 0 & 0 \\
x & 0 & 0
\end{array}\right), A_{3}(x)=\left(\begin{array}{ccc}
0 & x & 0 \\
-x^{*} & 0 & 0 \\
0 & 0 & 0
\end{array}\right)
$$

They act on $\mathfrak{J}$ through the commutators

$$
\tilde{A}_{i}\left(e_{a}\right) X=\frac{1}{2}\left[A_{i}\left(e_{a}\right), X\right], \quad i=1,2.3, \quad a=0,1, \ldots, 7 .
$$

The derivation algebra $\mathfrak{f}_{4(4)}$ of $\mathcal{H}_{3}\left(\mathbb{O}_{3}\right)$ is obtained from here by substituting the action of $\mathfrak{g}_{2}$ by that of $\mathfrak{g}_{2(2)}$ and by replacing $A_{i}\left(e_{a}\right)$ by $A_{i}\left(\tilde{e}_{a}\right)$ for $a=3,6,5,7$. It is for this non-compact form $\mathfrak{f}_{4(4)}$, that the Cartan elements are represented by real diagonal matrices in the isotropic basis $\xi_{\mu}^{\epsilon}$ (29)

$$
\begin{aligned}
& \alpha_{1}^{\vee}=H_{12}\left(=\alpha_{1}\right), \quad \alpha_{2}^{\vee}=H_{23}\left(=\alpha_{2}\right), \quad \alpha_{3}^{\vee}=2 H_{3}\left(=2 \alpha_{3}\right) \\
& \alpha_{0}^{\vee}=H_{01}-H_{2}-H_{3}=2 \alpha_{0} .
\end{aligned}
$$


The physical meaning of the $\mathrm{F}_{4}$ covariance of the algebra $\mathfrak{J}$ is revealed by exhibiting the action of

$$
\mathrm{F}_{4}^{\omega}=\frac{\mathrm{SU}(3) \times \mathrm{SU}(3)}{\mathbb{Z}_{3}} \subset \mathrm{F}_{4}
$$

(one of the maximal subgroup according to the Borel-de Siebentahal theory on $\mathcal{H}_{3}(\mathbb{O})$ ). To do that we shall first extend the splitting of the octonions $\mathbb{O}=\mathbb{C} \oplus \mathbb{C}^{3}$ into a splitting of the exceptional Jordan algebra, $H_{3}(\mathbb{O})=H_{3}(\mathbb{C}) \oplus \mathbb{C}[3]$

$$
\mathcal{H}_{3}(\mathbb{O}) \ni X(\xi, x)=\left(\begin{array}{lll}
\xi_{1} & x_{3} & x_{2}^{*} \\
x_{3}^{*} & \xi_{2} & x_{1} \\
x_{2} & x_{1}^{*} & \xi_{3}
\end{array}\right)=X(\xi, a)+X(0, \mathbf{z e})
$$

where

$$
\begin{aligned}
X(\xi, a) & =\left(\begin{array}{ccc}
\xi_{1} & a_{3} & \bar{a}_{2} \\
\bar{a}_{3} & \xi_{2} & a_{1} \\
a_{2} & \bar{a}_{1} & a_{3}
\end{array}\right) \\
a_{r} & =x_{r}^{0}+x_{r}^{7} e_{7}, \quad \bar{a}_{r}=x_{r}^{0}-x_{r}^{7} e_{7}, \quad r=1,2,3 \\
X(0, \mathbf{z e}) & =\left(\begin{array}{ccc}
0 & \mathbf{z}_{3} \mathbf{e}-\mathbf{z}_{\mathbf{2}} \mathbf{e} \\
-\mathbf{z}_{\mathbf{3}} \mathbf{e} & 0 & \mathbf{z}_{\mathbf{1}} \mathbf{e} \\
\mathbf{z}_{\mathbf{2}} \mathbf{e}-\mathbf{z}_{\mathbf{1}} \mathbf{e} & 0
\end{array}\right) \\
\mathbf{z}_{\mathbf{r}} \mathbf{e} & =z_{r}^{1} e_{1}+z_{r}^{2} e_{2}+z_{r}^{4} e_{4}, \quad z_{r}^{j}=x_{r}^{j}+x_{r}^{3 j(\bmod 7)} e_{7}
\end{aligned}
$$

in which we have used the conjugation property $(\mathbf{z e})^{*}=-\mathbf{z e}$ of imaginary octonions. Multiplications mixes the two terms in the right hand side of (91). The Freudenthal product $X(\xi, x) \times Y(\eta, b)$ can be expressed in a nice compact way if we substitute the skew symmetric octonionic matrices $X(0, \mathbf{z e}), X(0, \mathbf{w e})$ by $3 \times 3$ complex matrices $Z, W$

$$
X(0, \mathbf{z e}) \longleftrightarrow Z=\left(z_{r}^{j}, r=1,2,3, \jmath=1,2,4\right) \in \mathbb{C}[3]
$$

which transform naturally under the subgroup (90).

Indeed, using the fact that the matrices $X(0, \mathbf{z e})$ and $X(0, \mathbf{w e})$ are traceless their Fredenthal product (78) simplifies and we find

$$
\begin{gathered}
X(\xi, a) \quad \times X(0, \mathbf{w e})=X(\xi, a) \circ X(0, \mathbf{w e})-\frac{\xi_{1}+\xi_{2}+\xi_{3}}{2} X(0, \mathbf{w e}) \\
\Longrightarrow X(\xi, a) \times W=-\frac{1}{2} W X(\xi, a), \text { for } W=\left(w_{r}^{j}\right)
\end{gathered}
$$




$$
\begin{aligned}
& X(0, \mathbf{z e}) \times X(0, \mathbf{w e})=X(0, \mathbf{z e}) \circ X(0, \mathbf{w e})-\frac{1}{2} \operatorname{tr}(X(0, \mathbf{z e}) X(0, \mathbf{w e}) E) \\
& X(0, \mathbf{z e}) \times X(0, \mathbf{w e}) \leftrightarrow-\frac{1}{2}\left(W^{*} Z+Z^{*} W+\bar{Z} \times \bar{W}\right)
\end{aligned}
$$

where $Z \times W=\left(\epsilon_{r s t}\left(\mathbf{z}_{s} \times \mathbf{w}_{t}\right)^{j}\right)$, so that

$$
\begin{aligned}
(X(\xi, a) & +Z) \times(X(\eta, b)+W)=X(\zeta, c)+V \\
X(\zeta, c) & =X(\xi, a) \times X(\eta, b)-\frac{1}{2}\left(Z^{*} W-W^{*} Z\right) \\
V & =-\frac{1}{2}(W X(\xi, a)+Z X(\eta, b)+\bar{Z} \times \bar{W}) .
\end{aligned}
$$

Thus, if we set $V=\left(v_{r}^{j}\right)$ we would have

$$
\begin{aligned}
& 2 \mathbf{v}_{\mathbf{1}}=-\xi_{1} \mathbf{w}_{\mathbf{1}}-\bar{a}_{3} \mathbf{w}_{\mathbf{2}}-a_{2} \mathbf{w}_{\mathbf{3}}-\overline{\mathbf{z}}_{\mathbf{2}} \times \overline{\mathbf{w}}_{\mathbf{3}} \\
& 2 \mathbf{v}_{\mathbf{2}}=-a_{3} \mathbf{w}_{\mathbf{1}}-\xi_{2} \mathbf{w}_{\mathbf{2}}-\bar{a}_{1} \mathbf{w}_{\mathbf{3}}-\overline{\mathbf{z}}_{\mathbf{3}} \times \overline{\mathbf{w}}_{\mathbf{1}} \\
& 2 \mathbf{v}_{\mathbf{3}}=-\bar{a}_{2} \mathbf{w}_{1}-a_{1} \mathbf{w}_{2}-\xi_{3} \mathbf{w}_{3}-\overline{\mathbf{z}}_{\mathbf{1}} \times \overline{\mathbf{w}}_{\mathbf{2}} .
\end{aligned}
$$

The inner product in $\mathfrak{J}$ is express in terms of the components $X(\xi, a)$ and $Z$ as

$$
\begin{aligned}
(X, Y) & =\operatorname{tr} X \circ Y=(X(\xi, a), X(\eta, b))+2(Z, W) \\
2(Z, W) & =\operatorname{Tr}\left(Z^{*} W+W^{*} Z\right)=2 \sum_{r=1}^{3} \sum_{j=1,2,4}\left(\bar{z}_{r}^{j} w_{r}^{j}+\bar{w}_{r}^{j} z_{r}^{j}\right) .
\end{aligned}
$$

In the applications to the Standard Model of particle physics the (upper) index $j$ of $z(j=1,2,4)$ labels quark's colour while $r \in\{1,2,3\}$ is a family (or flavour) index. The $\mathrm{SU}(3)$ subgroup of $\mathrm{G}_{2}$, displayed in Section 2 acting on individual (imaginary) octonions is the colour group.

The subgroup $\mathrm{F}_{4}^{\omega}$ (cf. (90)) is defined as the commutant of the automorphism $\omega$ of order three in $\mathrm{F}_{4}$

$$
\begin{aligned}
\omega X(\xi, x) & =\left(\begin{array}{ccc}
\xi_{1} & \omega x_{3} & \left(\omega x_{2}\right)^{*} \\
\left(\omega x_{3}\right)^{*} & \xi_{2} & \omega x_{1} \\
\omega x_{2} & \left(\omega x_{1}\right)^{*} & \xi_{3}
\end{array}\right), \quad \omega(a+\mathbf{z e})=a+\omega_{7} \mathbf{z e} \\
\omega(X(\xi, a)+Z) & =X(\xi, a)+\omega_{7} Z, \quad \omega_{7}=-\frac{1}{2}+\frac{\sqrt{3}}{2} e_{7}, \quad \omega_{7}^{3}=1=\omega^{3} .
\end{aligned}
$$

We leave to the reader to verify that the restriction of $\omega$ to $\mathbb{O}$, given by

$$
\omega x=\omega(a+\mathbf{z e})=a+\omega_{7} \mathbf{z e}
$$


is an automorphism of $\mathbb{O}$ and that its commutant in $\mathrm{G}_{2}$ is $\mathrm{SU}(3)$. (One uses, in particular, the relation $\left.\omega_{7} \mathbf{z e} \omega_{7}=\omega_{7} \bar{\omega}_{7} \mathbf{z e}=\mathbf{z e}\right)$. The automorphisms $\mathfrak{g} \in \mathrm{F}_{4}^{\omega}$ that commute with $\omega(98)$ are given by pairs $g=(A, U) \in \mathrm{SU}(3) \times \mathrm{SU}(3)$ acting on $\mathcal{H}_{3}(\mathbb{O})$ by

$$
(A, U)(X(\xi, a)+Z)=A X(\xi, a) A^{*}+U Z A^{*} .
$$

The central subgroup

$$
\mathbb{Z}_{3}=\left\{(1,1),\left(\omega_{7}, \omega_{7}\right),\left(\omega_{7}^{2}, \omega_{7}^{2}\right)\right\} \in \mathrm{SU}(3) \times \mathrm{SU}(3)
$$

acts trivially on $\mathcal{H}_{3}(\mathbb{O})$. We see that unitary matrix $U$ acts (in (98)) on the colour index $j$ and hence belongs to the (unbroken) colour group $\mathrm{SU}(3)_{\mathrm{c}}$, while $A$ spans the (badly broken) family symmetry.

\subsection{The Jordan Subalgebra $\mathrm{JSpin}_{9}$ of $\mathcal{H}_{3}(\mathbb{O})$ and its Automorphism Group $\operatorname{Spin}(9) \subset \mathrm{F}_{4}$}

The ten dimensional Jordan algebra JSpin ${ }_{9}$ can be identified with the algebra of $2 \times$ 2 hermitian octonionic matrices $\mathcal{H}_{2}(\mathbb{O})$ equipped with the Jordan matrix product. It is generated by the 9 -dimensional vector subspace $s \mathcal{H}_{2}(\mathbb{O})$ of traceless matrices of $\mathcal{H}_{2}(\mathbb{O})$ whose square is, in fact, a positive real scalar

$$
X=\left(\begin{array}{cc}
\xi & x \\
x^{*}-\xi
\end{array}\right) \Rightarrow X^{2}=\left(\xi^{2}+x^{*} x\right) 11, \quad x \in \mathbb{O}, \quad \xi \in \mathbb{R} .
$$

$\mathrm{JSpin}_{9}$ is a (special) Jordan subalgebra of the (associative) matrix algebra $\mathbb{R}\left[2^{4}\right]$ that provides an IR of Cliff 9 . Clearly, it is a subalgebra of $\mathcal{H}_{3}(\mathbb{O})$-consisting of $3 \times$ 3 matrices with vanishing first row and first column. Its automorphism group is the subgroup $\operatorname{Spin}(9) \subset \mathrm{F}_{4}$ which stabilizes the projector $E_{1}: \operatorname{Spin}(9)=\left(\mathrm{F}_{4}\right)_{\mathrm{E}_{1}} \subset$ $\mathrm{F}_{4}$.

With our interpretation of $\mathfrak{J}=\mathcal{H}_{3}(\mathbb{O})$ as (possibly part of) the finite quantum algebra of the Standard Model of particle physics $\mathrm{JSpin}_{9}$ is the subalgebra corresponding to the first generation of (left chiral) quarks and leptons

$$
\left(\begin{array}{cc}
\nu_{L} & u_{L}^{j} \\
e_{L}^{-} & d_{L}^{j}
\end{array}\right), \quad j \quad \text { is the colour index. }
$$

They are physically distinguished as being much lighter than the particles in the second and third generation and therefore the relevant ones for the low energy physics. This justifies a more detailed study of the Jordan spin factor $\mathrm{JSpin}_{9}$ and its symmetry group. 
To begin with we shall interpret the 16-dimensional (real) spinor representation $S_{9}$ of $\operatorname{Spin}(9)$ as describing the two four-dimensional complex representations of the first generation of particles. They correspond to the splitting of $S_{9}$ into two 8-dimensional spinor representations $S_{8}^{\uparrow}$ and $S_{8}^{\downarrow}$ of $\operatorname{Spin}(8)$ that appear as eigenvectors of the Coxeter element $\omega_{8}$ of $\mathrm{Cl}_{8}$ (see Table 1)

$$
S_{9}=S_{8}^{\uparrow} \oplus S_{8}^{\downarrow}, \quad\left(\omega_{8}-1\right) S_{8}^{\uparrow}=0=\left(\omega_{8}+1\right) S_{8}^{\downarrow} .
$$

In fact, $\mathrm{Cl}_{9}$ is isomorphic (as ungraded algebra) to the direct sum of two $16 \times 16$ matrix algebras

$$
C l_{9} \approx \mathbb{R}\left[2^{4}\right] \oplus \mathbb{R}\left[2^{4}\right] .
$$

Each of the irreducible components is spanned by Cliff $_{8}$. Here is a (real) basis of $\mathrm{Cliff}_{8} \Gamma$-matrices with diagonal $\omega_{8}$

$$
\begin{array}{lll}
\Gamma_{0}=\sigma_{1} \otimes P_{0}, & \Gamma_{a}=c \otimes P_{a}, & P_{0}=\mathbb{1}_{8}=\mathbb{1} \otimes \mathbb{1} \otimes \mathbb{1} \\
P_{1}=\mathbb{1} \otimes c \otimes \sigma_{3}, & P_{2}=c^{*} \otimes \sigma_{3} \otimes \sigma_{3}, & P_{4}=\sigma_{1} \otimes c^{*} \otimes \sigma_{1} \\
P_{3}=\sigma_{3} \otimes c^{*} \otimes \sigma_{1}, & P_{6}=c \otimes \mathbb{1} \otimes \sigma_{1}, & P_{5}=c^{*} \otimes \sigma_{1} \otimes \sigma_{3} \\
P_{7}=\mathbb{1} \otimes \mathbb{1} \otimes c^{*}, & a=1, \ldots, 7 & \\
\Gamma_{8} \equiv \omega_{8}=\Gamma_{0} \Gamma_{1} \Gamma_{2} \Gamma_{3} \Gamma_{4} \Gamma_{5} \Gamma_{6} \Gamma_{7}=\sigma_{3} \otimes \mathbb{1} \otimes \mathbb{1} \otimes \mathbb{1}=\sigma_{3} \otimes \omega_{-7} \\
c^{*}=-c=\left(\begin{array}{rr}
0 & -1 \\
1 & 0
\end{array}\right) .
\end{array}
$$

(Each factor 11 in (104) is a $2 \times 2$ matrix.) The $\left(\begin{array}{l}9 \\ 2\end{array}\right)=36$ generators of the Lie algebra $\operatorname{Spin}(9)$ can be chosen as the commutators of these matrices. The maximal Lie subalgebra $\mathfrak{s u}(4) \oplus \mathfrak{s u}(2)$ of $\operatorname{Spin}(9)$ is spanned by

$$
\begin{aligned}
\mathfrak{s u}(4):\left\{\Gamma_{a b}\right. & \left.=\frac{1}{2}\left[\Gamma_{a}, \Gamma_{b}\right]=\Gamma_{a} \Gamma_{b} \quad \text { for } \quad a<b, \quad a, b=1, \ldots, 6\right\} \\
\mathrm{i} \mathfrak{s u}(2): \quad 2 I_{1} & =\mathrm{i} \Gamma_{7} \omega_{8}=\sigma_{1} \otimes \mathbb{1} \otimes \mathbb{1} \otimes \sigma_{2}, 2 I_{2}=\mathrm{i} \Gamma_{0} \omega_{8}=\sigma_{2} \otimes \mathbb{1} \otimes \mathbb{1} \otimes \mathbb{1} \quad \text { (106) } \\
2 I_{3} & =\mathrm{i} \Gamma_{0} \Gamma_{7}=\sigma_{3} \otimes \mathbb{1} \otimes \mathbb{1} \otimes \sigma_{2}, \quad\left[I_{1}, I_{2}\right]=\mathrm{i} I_{3}, \quad\left[I_{j}, \Gamma_{a b}\right]=0 .
\end{aligned}
$$

The general element $X(101)$ of the space $s \mathcal{H}_{2}(\mathbb{O})$ is written as

$$
X=\xi \omega_{8}+x^{a} \Gamma_{a}=\left(\xi \sigma_{3}+x^{0} \sigma_{1}\right) \otimes P_{0}+c \otimes \sum_{a=1}^{7} x^{a} P_{a} .
$$

The $8 \otimes 8$ matrices $P_{a}$ are an 8 -dimensional counterpart of the $2 \otimes 2$ matrix realization of the quaternion units $1, q_{j}=-\mathrm{i} \sigma_{j}$. They are characterized by similar anticommutation relations and product of imaginary units

$$
\begin{array}{rlrl}
P_{a} P_{b}^{*}+P_{b} P_{a}^{*} & =2 \delta_{a b}, \quad & a, b=0,1, \ldots, 7 & \\
P_{\bar{a}}^{*} & =-P_{\bar{a}}, \quad \bar{a}=1, \ldots, 7, \quad P_{1} \ldots P_{7}=\omega_{-7}=1
\end{array}
$$


while the 28 skew symmetric matrices $\frac{1}{2}\left(P_{a} P_{b}^{*}-P_{b} P_{a}^{*}\right)$ span the Lie algebra $\mathfrak{s o}(8)$.

We stress that the map $e_{a} \rightarrow P_{a}$ (unlike the representation $q_{j}=-\mathrm{i} \sigma_{j}$ ) only respects the Jordan products (i.e. the anticcommutators) of the octonion units, not their commutators. It could not have been otherwise as the $P_{a}$ belong to the 64dimensional associative algebra of real $8 \times 8$ matrices which can be identified (due to the last relation (108)) with the Clifford algebra Cliff $_{-6}$.

The correspondence between octonions $x=x^{a} e_{a}$ and $8 \times 8$ matrices $\hat{x}=x^{a} P_{a}$ is norm preserving

$$
x=x^{a} e_{a} \longleftrightarrow \hat{x}=x^{a} P_{a} \quad \Longrightarrow \quad x x^{*}=N(x)=\hat{x} \hat{x^{*}} .
$$

The norm preserving action of the Lie algebra $\mathfrak{s o}(8)$ on $x$ given by the operators $G_{a b}$ (51) corresponds to commutation with $\frac{1}{2} \hat{G}_{a b}$ where

$$
\begin{array}{lll}
\hat{G}_{a b}=\frac{1}{2}\left(P_{a} P_{b}^{*}-P_{b} P_{a}^{*}\right), \quad \text { i.e., } & \hat{G}_{a 0}=P_{a}=-\hat{G}_{0 a} \\
\hat{G}_{a b}=-P_{a b}=P_{b a} \quad\left(=-\hat{G}_{b a}\right) & \text { for } \quad 0<a<b .
\end{array}
$$

We have

$$
\frac{1}{2}\left[\hat{G}_{a b}, \hat{x}\right]=P_{a} x_{b}-x_{a} P_{b} \quad \longleftrightarrow \quad G_{a b} x=e_{a} x_{b}-x_{a} e_{b} .
$$

From now on we shall omit the hat on $\hat{G}_{a b}$ and will identify it with $G_{a b}$. The Lie subalgebra $\mathfrak{s u}(4)=\mathfrak{s o}(6)$ is spanned by $G_{a b}, a, b=1, \ldots, 6$. Its commutant in $\mathfrak{s o}(8)$ consists of the multiples of the $\mathrm{U}(1)$ generator $G_{07}$, that corresponds to the third component of the weak isospin $I_{3}$ (106). In order to reveal the physical meaning of the $\mathfrak{s u}(4)$ Lie algebra we shall identify its colour $\mathfrak{s u}(3)$ subalgebra which belongs to $\mathfrak{g}_{2} \subset \mathfrak{s o}(7)$. According to the results of Section 2.3 it is spanned by

$$
\begin{aligned}
& -\mathrm{i} H_{12}=G_{13}-G_{26}, \quad-\mathrm{i} H_{24}=G_{26}-G_{45} \\
& L_{12}=G_{12}+G_{36}, \quad L_{24}=G_{24}+G_{65}, \quad L_{14}=G_{14}+G_{35} \\
& N_{12}=G_{16}+G_{23}, \quad N_{24}=G_{25}+G_{46}, \quad N_{14}=G_{15}+G_{43} \text {. }
\end{aligned}
$$

The commutant $\mathfrak{u}(1)$ of $\mathfrak{s u}(3)$ in $\mathfrak{s u}(4)$ is spanned by the Cartan element

$$
\mathrm{i}\left(h_{1}+h_{2}+h_{3}\right)=G_{13}+G_{26}+G_{45}
$$

whose physical meaning will be made clear shortly. The operators $G_{13}, G_{26}$ and $G_{45}$ form a basis of the Cartan subalgebra of $\mathfrak{s o}(6)$

$$
\begin{aligned}
G_{13} & =-P_{1} P_{3}=\sigma_{3} \otimes \mathbb{1} \otimes c^{*}, \quad G_{26}=\mathbb{1} \otimes \sigma_{3} \otimes c^{*} \\
G_{45} & =\sigma_{3} \otimes \sigma_{3} \otimes c^{*} \Longrightarrow G_{13} G_{26} G_{45}=\omega_{6}=\mathbb{1} \otimes \mathbb{1} \otimes c=P_{7}^{*} \\
w_{-6} & =P_{1} P_{2} P_{3} P_{4} P_{5} P_{6}=P_{1} P_{3}^{*} P_{2} P_{6}^{*} P_{4} P_{5}^{*} .
\end{aligned}
$$


The equation $\omega_{-6}=P_{7}^{*}$ and $\omega_{-7}=\omega_{-6} P_{7}$ ) imply the last equation (108). The resulting real 8-dimensional representation of $\mathfrak{s u}(4)$ (given by the matrices $\hat{G}_{a b}=$ $G_{a b}(110)$ for $\left.1 \leq a, b \leq 6\right)$ is equivalent to a complex four-dimensional representation which can be obtained from (110) by identifying the matrix $P_{7}=\mathbb{1} \otimes \mathbb{1} \otimes c^{*}$ (which commutes with the above $G_{a b}$ ) with the imaginary unit i. For the Cartan generators (115) we obtain, in particular

$$
\begin{gathered}
P_{7} \rightarrow \mathrm{i} \Longrightarrow G_{a b} \rightarrow \gamma_{a b}, \quad \gamma_{13}=\mathrm{i} \sigma_{3} \otimes \mathbb{1}, \quad \gamma_{26}=\mathrm{i} 11 \otimes \sigma_{3} \\
\gamma_{45}=\mathrm{i} \sigma_{3} \otimes \sigma_{3} \quad \longrightarrow \quad \gamma_{13} \gamma_{26} \gamma_{45}=-\mathrm{i} .
\end{gathered}
$$

We shall go instead in the opposite direction: diagonalizing the 16-dimensional hermitian Cartan matrices $h_{j}=\mathrm{i} \Gamma_{j 3 j}, j=1,2,4$, in a complexified basis in which $h_{j}$ become real diagonal. To this end we use the unitary similarity transformation

$$
\Gamma \rightarrow S \Gamma_{a} S^{*}, \quad S=\mathbb{1} \otimes \mathbb{1} \otimes \mathbb{1} \otimes \frac{1+\mathrm{i} \sigma_{1}}{\sqrt{2}} \Rightarrow S S^{*}=\mathbb{1}_{16}
$$

with the result

$$
\begin{aligned}
& h_{1}:=S i \Gamma_{13} S^{*}=-\mathbb{1} \otimes \sigma_{3} \otimes \mathbb{1} \otimes \sigma_{3} \\
& h_{2}:=S i \Gamma_{26} S^{*}=-\mathbb{1} \otimes \mathbb{1} \otimes \sigma_{3} \otimes \sigma_{3} \\
& h_{3}:=S i \Gamma_{45} S^{*}=-\mathbb{1} \otimes \sigma_{3} \otimes \sigma_{3} \otimes \sigma_{3} .
\end{aligned}
$$

Inserting the matrices in the tensor products, so that

$$
\mathbb{1} \otimes \sigma_{3}=\left(\begin{array}{cc}
\sigma_{3} & 0 \\
0 & \sigma_{3}
\end{array}\right), \quad \sigma_{3} \otimes \mathbb{1}=\left(\begin{array}{cc}
\mathbb{1} & 0 \\
0 & -\mathbb{1}
\end{array}\right), \quad \sigma_{3} \otimes \sigma_{3}=\left(\begin{array}{cc}
\sigma_{3} & 0 \\
0 & -\sigma_{3}
\end{array}\right)
$$

we find for the sum (113) the product of the $2 \times 2$ unit matrix with a $4 \times 4$ diagonal matrix with $2 \times 2$ diagonal bloks

$$
h_{1}+h_{2}+h_{4}=\mathbb{1} \otimes\left(\begin{array}{cccc}
-3 \sigma_{3} & 0 & 0 & 0 \\
0 & \sigma_{3} & 0 & 0 \\
0 & 0 & \sigma_{3} & 0 \\
0 & 0 & 0 & \sigma_{3}
\end{array}\right) .
$$

\subsection{The Structure Group $\operatorname{Spin}(9,1)$ of $\mathrm{JSpin}_{9}$ and its 32-Dimensional Dirac Spinor Representation}

The spectrum of the operator $h_{1}+h_{2}+h_{4}$ consisting of four eigenvalues \pm 3 and \pm 1 corresponds to the odd part of the operator $3 Y$, where $Y$ is the weak hypercharge acting on the 32-dimensional space of fundamental fermions and antifermions of 
the Standard Model (SM). This motivates us to go one step further, to the structure group $\operatorname{Spin}(9,1)$ of the Jordan spin factor $\mathrm{JSpin}_{9}$ which, by definition, preserves the determinant

$$
\operatorname{det}\left(\begin{array}{cc}
\xi_{1} & x \\
x^{*} & \xi_{2}
\end{array}\right)=\xi_{1} \xi_{2}-x x^{*}, \quad \xi_{1,2} \in \mathbb{R}, x \in \mathbb{O} .
$$

Its Lie algebra is spanned by the $32 \times 32$ matrices

$$
\begin{aligned}
T_{a b} & =\frac{1}{2}\left[T_{a}, T_{b}\right] \quad \text { for } \quad a, b=-1,0,1, \ldots, 8 \\
T_{a} & =\sigma_{1} \otimes S \Gamma_{a} S^{*}, \quad a=0,1, \ldots, 8, \quad \Gamma_{8}=\omega_{8}, \quad T_{-1}=c \otimes \mathbb{1}_{16}
\end{aligned}
$$

with $T_{a}$ being the generators of Cliff $(9,1)$. It is another real form of the complexification of $\mathfrak{s o}(10)$, the ultimate "grand unified" Lie algebra (see [4] for a pedagogical review and references). The (complexified) Cartan subalgebra of $\mathfrak{s o}(9,1)$ is spanned by five diagonal matrices

$$
\begin{aligned}
H_{j} & =\mathbb{1} \otimes h_{j}=\mathrm{i} T_{j 3 j}, \quad j=1,2,4, \quad H_{0}=\mathrm{i} T_{07} \\
H_{8} & =T_{-18}=\sigma_{3} \otimes \sigma_{3} \otimes \mathbb{1}_{8} \\
\gamma & =H_{0} H_{1} H_{2} H_{4} H_{8}=-\sigma_{3} \otimes \mathbb{1}_{16}=-\omega_{9,1} .
\end{aligned}
$$

Here $\gamma$ is the parity operator taking value 1 for the left chiral fermions and -1 for the right chiral fermions. The fundamental fermions are labeled by three quantum numbers: the parity $\gamma$, the third component $I_{3}$ of the weak isospin and the weak hypercharge $Y$ defined as eigenvalue of the corresponding operators

$$
\begin{aligned}
& 2 I_{3}=\frac{1}{2}\left(H_{8}-H_{0}\right)=\operatorname{diag}\left\{\left(\sigma_{3}^{-}\right)^{\times 4},\left(-\sigma_{3}^{-}\right)^{\times 4},\left(-\sigma_{3}^{+}\right)^{\times 4},\left(\sigma_{3}^{+}\right)^{\times 4}\right\} \\
& \sigma_{3}^{-}=\left(\begin{array}{ll}
0 & 0 \\
0 & 1
\end{array}\right), \quad \sigma_{3}^{+}=\left(\begin{array}{ll}
1 & 0 \\
0 & 0
\end{array}\right)
\end{aligned}
$$

where $\sigma^{\times 4}$ stands for a $4 \times 4$ block diagonal matrix with $2 \times 2$ matrix blocks $\sigma$ on the diagonal, and

$$
\begin{aligned}
& 3 Y=\frac{3}{2}\left(H_{8}+H_{0}\right)+H_{1}+H_{2}+H_{4}=\left(\begin{array}{ll}
A & 0 \\
0 & \tilde{A}
\end{array}\right) \\
& A=\operatorname{diag}\left\{\left(\begin{array}{ll}
0 & 0 \\
0 & 3
\end{array}\right), \quad\left(\begin{array}{rr}
4 & 0 \\
0 & -1
\end{array}\right)^{\times 3}, \quad\left(\begin{array}{rr}
-6 & 0 \\
0 & 3
\end{array}\right), \quad\left(\begin{array}{rr}
-2 & 0 \\
0 & -1
\end{array}\right)^{\times 3}\right\} \\
& \tilde{A}=\operatorname{diag}\left\{\left(\begin{array}{rr}
-3 & 0 \\
0 & 0
\end{array}\right), \quad\left(\begin{array}{rr}
1 & 0 \\
0 & -4
\end{array}\right)^{\times 3}, \quad\left(\begin{array}{rr}
-3 & 0 \\
0 & 6
\end{array}\right), \quad\left(\begin{array}{ll}
1 & 0 \\
0 & 2
\end{array}\right)^{\times 3}\right\} .
\end{aligned}
$$


The 16 fundamental particles of negative parity $(\gamma=-1)$ corresponding to right chiral fields, are characterized by the pairs of eigenvalues $\left(2 I_{3}, 3 Y\right)$ as follows

$$
\begin{aligned}
& \nu_{R} \backsim(0,0), \quad e_{R}^{+} \backsim(1,3), \quad u_{R} \backsim(0,4), \quad \bar{u}_{R} \backsim(-1,-1) \\
& \bar{d}_{R} \backsim(1,-1), \quad e_{R}^{-} \backsim(0,-6), \quad \bar{\nu}_{R} \backsim(-1,3), \quad d_{R} \backsim(0,-2) .
\end{aligned}
$$

The corresponding left chiral fermions $(\gamma=1)$ are obtained from (124) by going to antiparticles and changing the sign of the quantum numbers

$$
\begin{gathered}
\bar{\nu}_{L} \backsim(0,0), \quad e_{L}^{-} \backsim(-1,-3), \quad \bar{u}_{L} \backsim(0,-4), \quad u_{L} \backsim(1,1) \\
d_{L} \backsim(-1,1), \quad e_{L}^{+} \backsim(0,6), \quad \nu_{L} \backsim(1,-3), \quad \bar{d}_{L} \backsim(0,2) .
\end{gathered}
$$

The projection operators to this states are polynomial functions of the Cartan elements. The electric charge $Q$ is related to $Y$ by

$$
Q=I_{3}+\frac{1}{2} Y \quad \Rightarrow \quad 2 Q-Y \in \mathbb{Z}, \quad Q+Y \in \mathbb{Z} .
$$

We see that the 32-dimensional Dirac spinor splits into two 16-dimensional left and right Weyl spinors which transform under irreducible representations of $\operatorname{Spin}(9,1)$. The splitting between left and right (or, equivalently, between even and odd element of the Clifford algebra $\operatorname{Cliff}(5, \mathbb{C})$ ) thus appears to be more relevant also mathematically than the difference between particles and antiparticles.

\section{The Symmetry Algebra of the Standard Model}

It has been observed by Baez and Huerta [4] that the gauge group of the SM

$$
S(\mathrm{U}(2) \times \mathrm{SU}(3))=\frac{\mathrm{SU}(2) \times \mathrm{SU}(3) \times \mathrm{U}(1)}{\mathbb{Z}_{6}}
$$

can be obtained as the intersection of the Georgi-Glashow and Pati-Salam grand unified theory groups $\mathrm{SU}(5)$ and $(\mathrm{SU}(4) \times \mathrm{SU}(2) \times \mathrm{SU}(2)) / \mathbb{Z}_{2}$ viewed as subgroup of Spin(10). It was suggested in [30] that one can deduce the symmetry of the SM by applying the Borel-de Siebenthal theory to the automorphism group $\mathrm{F}_{4}$ of the exceptional Jordan algebra J.

Here we shall consider instead maximal rank subalgebras of the complexified Lie algebra $\mathbb{C} \otimes \mathfrak{s o}(9,1)$ of the structure group $\operatorname{Spin}(9,1)$ of $\mathrm{JSpin}_{9}$ which commute with the exact symmetry Lie algebra

$$
\mathfrak{s u}(3)_{c} \oplus \mathfrak{u}(1)_{Q}, \quad Q=I_{3}+\frac{1}{2} Y .
$$


The resulting superselected observables (in the terminology introduced in [31]) give rise to a three dimensional abelian algebra spanned by the operators $2 I_{3}, 3 Y$ and

$$
H=H_{1}+H_{2}+H_{4}=\mathbb{1} \otimes\left(h_{1}+h_{2}+h_{4}\right)=\mathbb{1}_{4} \otimes\left(\begin{array}{cccc}
-3 \sigma_{3} & 0 & 0 & 0 \\
0 & \sigma_{3} & 0 & 0 \\
0 & 0 & \sigma_{3} & 0 \\
0 & 0 & 0 & \sigma_{3}
\end{array}\right)
$$

(cf. (118)), which are all traceless with integer eigenvalues. In order to reveal the physical meaning of $H$ we shall recall the realization of the 32-dimensional fermionic Fock space $\mathcal{F}$ as the exterior algebra

$$
\Lambda \mathbb{C}^{5}=\bigoplus_{\nu=0}^{5} \Lambda^{\nu}, \quad \Lambda^{\nu}=\Lambda^{\nu} \mathbb{C}^{5}
$$

where

$$
\Lambda^{0}=\bar{\nu}_{L}, \quad \Lambda^{1}=e_{R}^{+}, \bar{\nu}_{R}, \quad d_{R}=\left(d_{R}^{c}\right)
$$

see [4, Section 2].

More generally, the $2^{n}$-dimensional vector space of the Clifford algebra Cliff $(n, \mathbb{C})$ is isomorphic to the exterior algebra $\Lambda \mathbb{C}^{n}$. If $\left(e_{1}, \ldots, e_{n}\right)$ is basis in $\Lambda^{1} \mathbb{C}^{n}$ we can turn $\Lambda \mathbb{C}^{n}$ into a Hilbert space by introducing the orthonormal basis

$$
1, e_{1}, \ldots, \quad e_{n}, \quad e_{i} \wedge e_{j}, \quad 1 \leq i<j \leq n, \ldots, \quad e_{1} \wedge \cdots \wedge e_{n} .
$$

There is an equivalent fermionic Fock space realization of $\Lambda \mathbb{C}^{n} \simeq \mathcal{F}$ in terms of $n$ creation and $n$ annihilation operators $a_{i}^{*}$ and $a_{j}$ acting on $\mathcal{F}$ such that

$$
a_{i}^{*} \Psi=e_{i} \wedge \Psi, \quad\left\langle a_{i} \Phi, \Psi\right\rangle=\left\langle\Phi, a_{i}^{*} \Psi\right\rangle \quad \Phi, \Psi \in \mathcal{F} .
$$

It follows from (133) that $\left(a_{i}, a_{j}^{*}\right)$ satisfy the canonical anticommutation onrelations

$$
\left[a_{i}, a_{j}\right]_{+}=0=\left[a_{i}^{*}, a_{j}^{*}\right]_{+}, \quad\left[a_{i}, a_{j}^{*}\right]_{+}=\delta_{i j} .
$$

Furthermore, if we identify the "vector" $1 \in \Lambda^{0} \mathbb{C}$ with the Fock space vacuum $|0\rangle$ we shall have

$$
a_{i}|0\rangle=0, \quad a_{j}^{*}|0\rangle=e_{j}, \quad a_{i}^{*} a_{j}^{*}|0\rangle=e_{i} \wedge e_{j}, \quad \text { etc. }
$$

Returning to the case $n=5$ with the identification (131) we introduce the operators $r^{*}=\left(r_{+}^{*}, r_{0}^{*}\right), d^{*}=\left(d_{c}^{*}\right)$ which create the states in $\Lambda^{1}$

$$
r_{+}^{*}|0\rangle=e_{R}^{+}, \quad r_{0}^{*}|0\rangle=\bar{\nu}_{R}, \quad d^{*}|0\rangle=d_{R}, \quad|0\rangle=\bar{\nu}_{L}
$$


with quantum numbers $\left(2 I_{3}, 3 Y, H\right)=(1,3,3),(-1,3,3),(0,-2,1)^{\times 3}$, respectively (and their annihilation counterparts $r, d)$. The eigenvalues $(3,3,1)^{\times 3}$ of $H$ are the same as those of the operator

$$
H=\left[d, d^{*}\right]=3-2 d^{*} d
$$

(where we skip the sum sign over the colour index in $d^{*} d$ ). We note that $H$ is $i$ times a generator of $\mathfrak{s o}(6)(\subset \mathfrak{s o}(9))$ but not of $\mathfrak{s u}(5)$, so the sum of its eigenvalues in $\Lambda^{1}$ does not vanish but its trace on the entire right and left sectors, separately, is zero

$$
(\operatorname{tr} H)_{\text {odd }}=\operatorname{tr}_{\Lambda^{1}} H+\operatorname{tr}_{\Lambda^{3}} H+\operatorname{tr}_{\Lambda^{5}} H=9-6-3=0 .
$$

Furthermore, in the 16 -dimensional subspace $\mathcal{F}_{1}$ of $\mathcal{F}$ spanned by 8 isotopic doublets

$$
\left(\begin{array}{c}
\nu_{L} \\
e_{L}^{-}
\end{array}\right), \quad\left(\begin{array}{c}
u_{L} \\
d_{L}
\end{array}\right), \quad\left(\begin{array}{c}
e_{R}^{+} \\
\bar{\nu}_{R}
\end{array}\right), \quad\left(\begin{array}{c}
\bar{d}_{R} \\
\bar{u}_{R}
\end{array}\right)
$$

in which $3 Y$ and $2 I_{3}$ have odd eigenvalues and actually belong to $\mathfrak{s o}(8)$

$$
(-1)^{3 Y}=(-1)^{2 I_{3}}=-1, \quad 3 Y=H, \quad 2 I_{3}=\mathrm{i} \Gamma_{07}, Y=B-L \in \mathcal{F}_{1}
$$

where $B$ and $L$ are the baryon and lepton numbers. The space $\mathcal{F}_{1}$ in fact coincides with the spinor representation of $\operatorname{Spin}(9)$. The eight fundamental left chiral particles and their (right chiral) antiparticles that take part in the weak interaction span (inequivalent) 8-dimensional irreducible representations of $\operatorname{Spin}(8)$. All (anti)particles in the doublets are characterized by the pairs of eigenvalues $\left(2 I_{3}, 3 Y\right)$ (cf. (124) and (125))

$$
\begin{aligned}
& \nu_{L} \backsim(1,-3), \quad e_{L}^{-} \backsim(-1,-3), \quad u_{L} \backsim(1,1), \quad d_{L} \backsim(-1,1) \\
& e_{R}^{+} \backsim(1,3), \quad \bar{\nu}_{R} \backsim(-1,3), \quad \bar{d}_{R} \backsim(1,-1), \quad \bar{u}_{R} \backsim(-1,-1) .
\end{aligned}
$$

\section{Outlook}

The idea that exceptional structures in mathematics should characterize the fundamental constituents of matter has been with us since the ancient Greeks first contemplated the Platonic solids. The octonions, the elements of the ultimate division algebra, have been linked to the Standard Model of particle physics ever since 
Günaydin and Gürsey related them to the coloured quarks around 1973. When the idea of a finite quantum geometry emerged $[9,15]$ it became natural to look for a role of the exceptional algebraic structures in such a context. A promising step in this direction was made by Dubois-Violette [14] who pointed out that the exceptional Jordan algebras $\mathfrak{J}=\mathcal{H}_{3}(\mathbb{O})$ with its three octonions and three real elements offers room to the three families of quarks and leptons along with three Majorana neutrinos.

The next step [30], continued in the present notes, puts more emphasis on the automorphism (and isometry) groups of the algebraic structures. We identify such basic observables as the weak hypercharge and isospin as elements of the subalgebra $\mathfrak{s o}(9,1)$ of the Lie algebra of the structure group $\mathrm{E}_{6(-26)}$ that preserves the determinant of the elements of $\mathfrak{J}$.

In fact, the exceptional Jordan algebra is intimately related to all exceptional Lie groups [5]. It will be interesting to reveal further the role of the structure group $\mathrm{E}_{6(-26)}$ and the conformal group $\mathrm{E}_{7(-25)}$ of $\mathfrak{J}$ in the physics of the Standard Model. We intend to return to this problem in future work.

Intriguingly, the basic doublets that participate in the weak interaction fit in the 16-dimensional spinor representation of the subgroup $\operatorname{Spin}(9)$ of the (compact) automorphism group $\mathrm{F}_{4}$ of the exceptional Jordan algebra which in turn splits into two 8-dimensional $\operatorname{Spin}(8)$ spinors corresponding to the eight fundamental particles and to their antiparticles, respectively.

\section{Acknowledgements}

Ivan Todorov thanks Michel Dubois-Violette for enlightening discussions. His work has been supported in part by Grant DFNI T02/6 of the Bulgarian National Science Foundation. Svetla Drenska's work has been supported by the Bulgarian National Science Fund DFNI E02/6.

\section{Application A. The Fano Plane of Imaginary Octonions}

The multiplication table for the seven octonionic imaginary units can be recovered from the following properties

$$
\begin{aligned}
e_{i}^{2} & =-1, \quad i=1, \ldots, 7, \quad e_{i} e_{j}=-e_{j} e_{i} \\
e_{i} e_{j} & =e_{k} \Rightarrow e_{i+1} e_{j+1}=e_{k+1}, \quad e_{2 i} e_{2 j}=e_{2 k}
\end{aligned}
$$

where indices are counted modulo seven, and a single relation of the type

$$
e_{1} e_{2}=e_{4}
$$


producing a quaternionic line. We have displayed on Fig. 1 the points $e_{i}$ as nonzero triples of homogeneous coordinates taking values 0 and 1 such that the product $e_{i} e_{j}$ (in clockwise order) is obtained by adding the coordinates $(a, b, c), a, b, c \in$ $\{0,1\}$, modulo two.

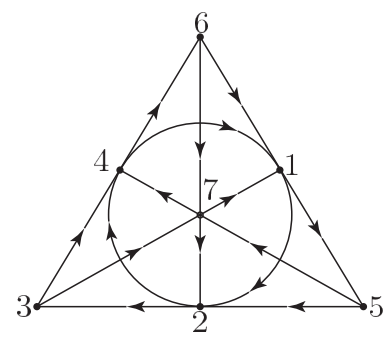

$$
\begin{gathered}
e_{1}=(0,0,1), e_{2}=(0,1,0) \Longrightarrow e_{1} e_{2}=e_{4}=(0,1,1) \\
e_{3}=(1,0,0) \Longrightarrow e_{2} e_{3}=e_{5}=(1,1,0) \\
e_{1} e_{5}=e_{6}=(1,1,1), \quad e_{4} e_{5}=e_{7}=(1,0,1) .
\end{gathered}
$$

Figure 1. Projective plane in $\mathbb{Z}_{2}^{3}$ with seven points and seven lines.

\section{Application B.}

Two bases of $\mathfrak{s o}(8)$ related by the outer automorphism $\pi$. The generators $G_{a b}$ of $\mathfrak{s o}(8)$ are given directly by their action on the octonion units (51)

$$
G_{a b} e_{b}=e_{a}, \quad G_{a b} e_{a}=-e_{b}, \quad G_{a b} e_{c}=0 \quad \text { for } \quad a \neq c \neq b .
$$

The action of $F_{a b}$ can also be deduced from definition (51) and multiplication rules:

$$
\begin{gathered}
F_{a b} e_{b}=\frac{1}{2} e_{a}, \quad F_{a b} e_{a}=-\frac{1}{2} e_{b}, \quad(a \neq b) F_{a b}=-F_{b a} \\
F_{j 0} e_{2 j}=\frac{1}{2} e_{4 j \bmod 7}\left(=-F_{0 j} e_{2 j}\right), \quad j=1,2,4 \\
F_{j 0} e_{3 j}=\frac{1}{2} e_{7}, \quad F_{70} e_{7}=\frac{1}{2} e_{3 j} \quad F_{07}=\frac{1}{2} e_{j} \\
F_{0 j} e_{6 j}=\frac{1}{2} e_{5 j}, \quad F_{j 0} e_{5 j}=\frac{1}{2} e_{6 j}, \quad\left[F_{j 0}, F_{0 k}\right]=F_{j k} .
\end{gathered}
$$


(All indices are counted mod7.) From (B.1) and (B.2) we find

$$
\begin{gathered}
2 F_{0 j}=G_{0 j}+G_{2 j 4 j}+G_{3 j 7}+G_{5 j 6 j} \\
2 F_{03 j}=G_{03 j}-G_{J 7}-G_{2 j 5 j}+G_{4 j 6 j}, \quad j=1,2,4 \\
2 F_{07}=G_{07}+G_{13}+G_{26}+G_{45} .
\end{gathered}
$$

In particular, taking the show symmetry of $G_{a b}$ and the counting mod7 into account we can write

$$
\begin{aligned}
& 2 F_{02}=G_{02}-G_{14}+G_{35}-G_{76} \\
& 2 F_{04}=G_{04}+G_{12}-G_{36}-G_{75} \\
& 2 F_{03}=G_{03}-G_{17}-G_{25}+G_{46} \\
& 2 F_{06}=G_{06}+G_{15}-G_{27}-G_{43} \\
& 2 F_{05}=G_{05}-G_{16}+G_{23}-G_{47} .
\end{aligned}
$$

Note that with the $a b c$ are ordering $(1,2,4,3,7,5,6)$ The first (positive) indices of $G(2,3,5 ; 1,3,7 ; 1,2,4)$ correspond to quaternionic triples: $e_{2} e_{3}=e_{5}, e_{1} e_{3}=e_{7}$, $e_{1} e_{2}=e_{4}$. Setting

$$
\begin{aligned}
G_{1}=\left(\begin{array}{l}
G_{01} \\
G_{24} \\
G_{37} \\
G_{56}
\end{array}\right), \quad G_{2}=\left(\begin{array}{l}
G_{02} \\
G_{14} \\
G_{35} \\
G_{76}
\end{array}\right), \quad G_{4}=\left(\begin{array}{l}
G_{04} \\
G_{12} \\
G_{36} \\
G_{75}
\end{array}\right), \quad G_{3}=\left(\begin{array}{l}
G_{03} \\
G_{17} \\
G_{25} \\
G_{46}
\end{array}\right) \\
G_{7}=\left(\begin{array}{l}
G_{07} \\
G_{13} \\
G_{26} \\
G_{45}
\end{array}\right), \quad G_{5}=\left(\begin{array}{l}
G_{05} \\
G_{16} \\
G_{23} \\
G_{47}
\end{array}\right), \quad G_{66}=\left(\begin{array}{l}
G_{15} \\
G_{27} \\
G_{43}
\end{array}\right)
\end{aligned}
$$

and similarly for $F_{1}, \ldots F_{6}$ we find

$$
\begin{gathered}
F_{a}=X_{a} G_{a}, \quad a=1, \ldots, 7, \quad \text { with } \\
X_{1}=X_{7}=\frac{1}{2}\left(\begin{array}{rrrr}
1 & 1 & 1 & 1 \\
1 & 1 & -1 & -1 \\
1 & -1 & 1 & -1 \\
1 & -1 & -1 & 1
\end{array}\right), \quad X_{2}=X_{5}=\frac{1}{2}\left(\begin{array}{rrrr}
1 & -1 & 1 & -1 \\
-1 & 1 & 1 & -1 \\
1 & 1 & 1 & 1 \\
-1 & -1 & 1 & 1
\end{array}\right) \\
X_{4}=X_{6}=\frac{1}{2}\left(\begin{array}{rrrr}
1 & 1 & -1 & -1 \\
1 & 1 & 1 & 1 \\
-1 & 1 & 1 & -1 \\
-1 & 1 & -1 & 1
\end{array}\right), \quad X_{3}=\frac{1}{2}\left(\begin{array}{rrrr}
1 & -1 & -1 & 1 \\
-1 & 1 & -1 & 1 \\
-1 & -1 & 1 & 1 \\
1 & 1 & 1 & 1
\end{array}\right) .
\end{gathered}
$$


They all define involutive transformations

$$
X_{k}^{2}=1, \quad \operatorname{det} X_{k}=-1, \quad k=1,2,3,4 .
$$

\section{References}

[1] Adams J., Lectures on Exceptional Lie Groups, Z. Mahmud and M. Mimira (Eds), Univ. Chicago Press, Chicago 1996.

[2] Atiyah M., Bott R. and Shapiro A., Clifford Modules, Topology 3 (1964) 338.

[3] Baez J., The Octonions, Bull. Amer. Math. Soc. 39 (2002) 145-205, Errata, ibid. 42 (2005) 213, math/0105155v4 [math.RA].

[4] Baez J. and Huerta J., The Algebra of Grand Unified Theory, Bull. Amer. Math. Soc. 47 (2010) 483-552, arXiv:0904.1556v2 [hep-th].

[5] Barton C. and Sudbery A., Magic Squares and Matrix Models of Lie Algebras, Adv. Math. 180 (2003) 596-647, math/0203010v2.

[6] van der Blij F., History of the Octaves, Simon Stevin Wis. Naturkundig Tidschrift 34E Jaargang Aflevering III (Februari 1961), pp 106-125.

[7] Borel A. and de Siebenthal J., Les Sous-Groupes Fermes de Rang Maximum des Groupes de Lie Clos, Comment. Math. Helv. 23 (1949) 200-221.

[8] Catto S., Gürcan Y., Khalfan A. and Kurt L., Unifying Ancient and Modern Geometries Through Octonions, J. Phys. Conf. Series 670 (2016) 012016.

[9] Connes A. and Lott J., Particle Models and Noncommutative Geometry, Nucl. Phys. Proc. Suppl. B 18 (1990) 29-47 .

[10] Dickson L., On Quaternions and their Generalization and the History of the Eight Square, Ann. Math. 20 (1919) 155-171.

[11] Dixon G., Division Algebras: Family Replication, J. Math. Phys. 45 (2004) 3878-3882.

[12] Dixon G., Division Algebras; Spinors; Idempotents; The Algebraic Structure of Reality, arXiv:1012.1304 [hep.th].

[13] Dixon G., Seeable Matter; Unseeable Antimatter, Comment. Math. Univ. Carolin. 55 (2014) 381-386; arXiv:1407.4818 [physics.gen-ph].

[14] Dubois-Violette M., Exceptional Quantum Geometry and Particle Physics, Nucl. Phys. B 912 (2016) 426-444, arXiv:1604.01247.

[15] Dubois-Violette M., Kerner R. and Madore J., Gauge Bosons in a NonCommutative Geometry, Phys. Lett. B 217 (1989) 485-488. 
[16] Farnswarth S. and Boyle L., 'Rethinking Connes' Approach to the Standard Model of Particle Physics via Non-Commutative Geometry, New J. Phys. 17 (2015) 023021, arXiv:1408.5367[hep-th].

[17] Freudenthal H., Lie Groups in the Foundation of Geometry, Adv. Math. 1 (1964) 145-190.

[18] Furey C., Standard Model Physics From an Algebra?, arXiv:1611.09182 [hep-th].

[19] Gilmore R., Lie Groups, Lie Algebras, and Some of Their Applications, Dover, New York 2008.

[20] Günaydin M., Pirron C. and Ruegg H., Moufang Plane and Octonionic Quantum Mechanics, Commun. Math. Phys. 61 (1978) 69-85.

[21] Gürsey F., Octonionic Structures in Particle Physics, Group Theoretical Methods in Physics, LNP 94, Springer, Berlin 1979, pp. 508-521, Gürsey F. and Tze C.-H., The Role of Division, Jordan and Related Algebras in Particle Physics, World Scintific, Singapore 1996.

[22] Iso S., Okada N. and Orikasa Y., Classically Conformal B - L Extended Standard Model, arXiv:0902.4050v3 [hep-ph].

[23] Jordan P., v. Neumann J. and Wigner E., On an Algebraic Generalization of the Quantum Mechanical Formalism, Ann. Math. 36 (1934) 29-64.

[24] Lounesto P., Clifford Algebras and Spinors, $2^{\text {nd }}$ Edn, London Math. Soc. Lecture Notes Series 286, Cambridge Univ. Press, Cambridge 2001.

[25] McCrimmon K., A Taste of Jordan Algebras, Springer, Berlin 2004.

[26] Roos G., Exceptional Symmetric Domains, In: Bruce Guilling et al (Eds), Symmetries in Complex Analysis, AMS, Contemporary Mathematics 468 (2008) 157-189, arXiv:0801.4076 [math.CV].

[27] Schafer R., Structure and Representations of nonassociative Algebras, Bull. Amer. Math. Soc. 61 (1955) 469-484, An Introduction to Nonassociative Algebras, Dover, New York 1995.

[28] Stoica O., The Standard Model Algebra, arXiv:1702.04336v2 [hep-th].

[29] Todorov I., Clifford Algebras and Spinors, Bulg. J. Phys. 58 (2011) 3-28, arXiv:1106.3197[math-ph].

[30] Todorov I. and Dubois-Violette M., Deducing the Symmetry of the Standard Model the Authomorphism and Structure Groups of the Exceptional Jordan Algebra, Bures-sur-Yvette preprint IHES/P/17/03.

[31] Wick J., Wightman A. and Wigner E., The Intrinsic Parity of Elementary Particles, Phys. Rev. 88 (1952) 101-105.

[32] Yokota I., Exceptional Lie Groups, arXiv:0902.0431. 
Ivan Todorov

Institute of Nuclear Research and Nuclear Energy

Bulgarian Academy of Sciences

72 Tzarigradsko Chaussee

Sofia 1784, BULGARIA

E-mail address: ivbortodorov@gmail.com

Svetla Drenska

Institute of Nuclear Research and Nuclear Energy

Bulgarian Academy of Sciences

72 Tzarigradsko Chaussee

Sofia 1784, BULGARIA

E-mail address: sdren@abv.bg 